\title{
Spheroidal forward modelling of the gravitational fields of 1 Ceres and the Moon
}

\author{
M. Šprlák ${ }^{\mathrm{a}, *}$, S.-C. Han ${ }^{\mathrm{a}}$, W.E. Featherstone ${ }^{\mathrm{b}}$ \\ ${ }^{a}$ School of Engineering, Faculty of Engineering and Built Environment, University of Newcastle, University \\ Drive, Callaghan, NSW 2308, Australia \\ ${ }^{b}$ School of Earth and Planetary Sciences, Curtin University of Technology, GPO Box U1987, Perth, WA 6845, \\ Australia
}

\begin{abstract}
A novel, explicit, and efficient forward modelling of the spheroidal harmonic spectra of external planetary gravitational fields is developed in this article. We introduce the oblate spheroidal coordinate system and derive the mathematical apparatus for the analysis of the spheroidal harmonic spectrum from the volumetric bulk density and geometry of a gravitating body. We discretise the volume integral and formulate a new and efficient numerical algorithm for the spheroidal forward modelling. We provide complete sets of recursions for calculating the associated Legendre functions of the first kind and their integrals in the Supplementary Material. We also develop a computer program that implements the numerical algorithm and we test its performance. For this purpose, we consider synthetic gravitational fields of 1 Ceres (a significantly flattened asteroid) and of the Moon (a nearly spherical body). These tests prove high numerical accuracy and applicability of the spheroidal forward modelling up to degree and order 2519. We finally apply our spheroidal forward modelling and its simpler spherical counterpart for computing global gravitational field models up to degree and order 2519 generated by realistic topographic mass distributions of 1 Ceres and of the Moon. These models are compared in the spatial and spectral domains to manifest an enhanced applicability of the spheroidal approach with respect to the spherical one. In particular, we show an extended convergence space when using the spheroidal forward modelling and the corresponding harmonic representation for the oblate 1 Ceres.
\end{abstract}

Keywords: Asteroid Ceres, Asteroids, surfaces, Moon, Moon, surface

\section{Introduction}

Forward modelling is used for gravitational field determination in geodesy, geophysics and planetary sciences. It is based on Newton's (1687) integral for the gravitational potential (e.g., Green, 1828; Kellogg, 1929, Chap. 3). The masses can be replaced by the product of volume and bulk density, thus allowing different geometries and density distributions to be considered.

In the first approximation, most planetary bodies resemble spheres. Accordingly, spherical forward modelling techniques have been employed to calculate the gravitational potential and its functionals in the spatial domain. This has been achieved by decomposing Newton's integral and its first-, second-, and third-order spatial derivatives into spherical prisms or spherical

*Tel.:+61 24921 8984, Fax: +61249216991

Email address: michal.sprlak@gmail.com (M. Šprlák) 
tesseroids (e.g., Anderson, 1976; Asgharzadeh et al., 2007; Heck and Seitz, 2007; Wild-Pfeiffer, 2008; Uieda et al., 2016; Deng and Shen, 2018; Fukushima, 2018). Other spatial forward modelling strategies have considered discretisations by point masses, line masses, right-rectangular prisms, or polyhedra (e.g., Pohánka, 1988; Grüninger, 1990; Werner and Scheeres, 1996; Nagy et al., 2000; D'Urso, 2013; Werner, 2017).

Alternatively, the gravitational potential of a spherical gravitating body can be parametrised by its spherical harmonic spectrum. Practical advantages of the spherical harmonic representation have resulted in numerous spectral forward modelling techniques (e.g., Lee and Kaula, 1967; Balmino et al., 1973; Lachapelle, 1976; Rummel et al., 1988; Pavlis and Rapp, 1990; Balmino, 1994; Wieczorek and Phillips, 1998; Wieczorek, 2007; Balmino et al., 2012; Grombein et al., 2016; Kuhn and Hirt, 2016; Rexer et al., 2016; Tenzer et al., 2016; Šprlák et al., 2018), which are based on various simplifications on the bulk density and geometry of the gravitating body.

Some planetary bodies, however, tend to be flattened at the poles and their shapes are closer to oblate spheroids (rotational ellipsoids) than spheres (e.g., Chandrasekhar, 1969; Moritz, 1990). This has motivated spheroidal (also known as ellipsoidal) formulations of Newton's integral, including its first- and second-order spatial derivatives (Ardalan and Safari, 2004; Novák and Grafarend, 2005; Roussel et al., 2015; Novák and Šprlák, 2018). Spectral approaches have also been proposed for the forward modelling of the spheroidal harmonic spectra (Wang and Yang, 2013; Hu and Jekeli, 2015; Reimond and Baur, 2016; Sebera et al., 2016).

An alternative to the existing spectral methods for oblate spheroidal bodies may comprise spherical modelling followed by transformation of the spherical harmonic spectrum to its spheroidal counterpart (e.g., Hotine, 1969, pp. 194-195; Jekeli, 1981). This pragmatic procedure, however, is not without difficulties, as discussed by (Lowes and Winch, 2012), and cannot completely substitute an explicit spheroidal forward modelling in the spectral domain, which is what we present here.

In this article, we develop, implement, test and demonstrate applicability of the forward modelling for spheroidal harmonic spectra. Our approach improves and differs from the existing techniques in several regards: 1) It allows for implementing 3D spatially variable density. In contrast, the method by Wang and Yang (2013) was developed with the assumption of only a laterally varying density distribution. 2) It provides the spheroidal harmonic spectrum by one volume integral. This differs from the hybrid methods by (Hu and Jekeli, 2015; Sebera et al., 2016), which combine two surface integrals for planetary bodies of constant density and discretised by polyhedra. 3) It presents an explicit discretisation of the integral representation by spheroidal tesseroids and, for regularly gridded data, we provide a numerically efficient algorithm based on the fast/discrete Fourier transform. 4) It uses simple recursion formulas, which are numerically more efficient than the Laurent series formalism derived by (Wang and Yang, 2013). 5) The former studies such as Wang and Yang (2013) demonstrated the spheroidal computation up to d/o 180 for the Earth, whereas our spheroidal implementation is demonstrated to be accurate up to ultra-high d/o 2519, and for the significantly flattened planetary body of 1 Ceres.

We also acknowledge studies on the forward modelling for the even more complex geometry of a tri-axial ellipsoid (e.g., Garmier and Barriot, 2001; Park et al., 2014; Hu and Jekeli, 2015; Reimond and Baur, 2016). These have been restricted to d/o less than $\sim 20$ due to numerical problems in calculating the corresponding basis functions, so will not be attempted here. 


\section{Forward modelling of the spheroidal harmonic spectrum}

In this Section, we summarise the fundamentals for the evaluation of the spheroidal harmonic spectra from the geometry and bulk density of a gravitating body (e.g., Jekeli, 1981; Moritz, 1990; Grafarend, 2015). As we consider only oblate spheroidal geometry, we use so-called planetocentric oblate spheroidal coordinates $u$ and $\Omega_{e}=(\beta, \lambda)$. In the geodetic literature, these are also called one-parametric Jacobi or mixed elliptic-trigonometric elliptic coordinates (Thong and Grafarend, 1989; Ardalan, 2000). However, in the mathematical and other literature, they are more commonly called ellipsoidal coordinates.

The coordinate triplet we use is defined by the semi-minor axis $u \in[0, \infty)$, the reduced latitude $\beta \in\left[-90^{\circ},+90^{\circ}\right]$, and the longitude $\lambda \in\left[0^{\circ},+360^{\circ}\right)$, see Fig. 1. If the geometry and density distribution are expressed in terms of spherical or geodetic coordinates, they can be transformed to $u$ and $\Omega_{e}=(\beta, \lambda)$ using closed-form algorithms (e.g., Featherstone and Claessens, 2008). The use of planetocentric oblate spheroidal coordinates is essential because separation of variables can be performed in this coordinate triplet when solving Laplace's equation (Moon and Spencer, 1953). Consequently, the gravitational potential can be represented by the spheroidal harmonic series of Eq. (5) that makes formulation of the spectral forward modelling possible.

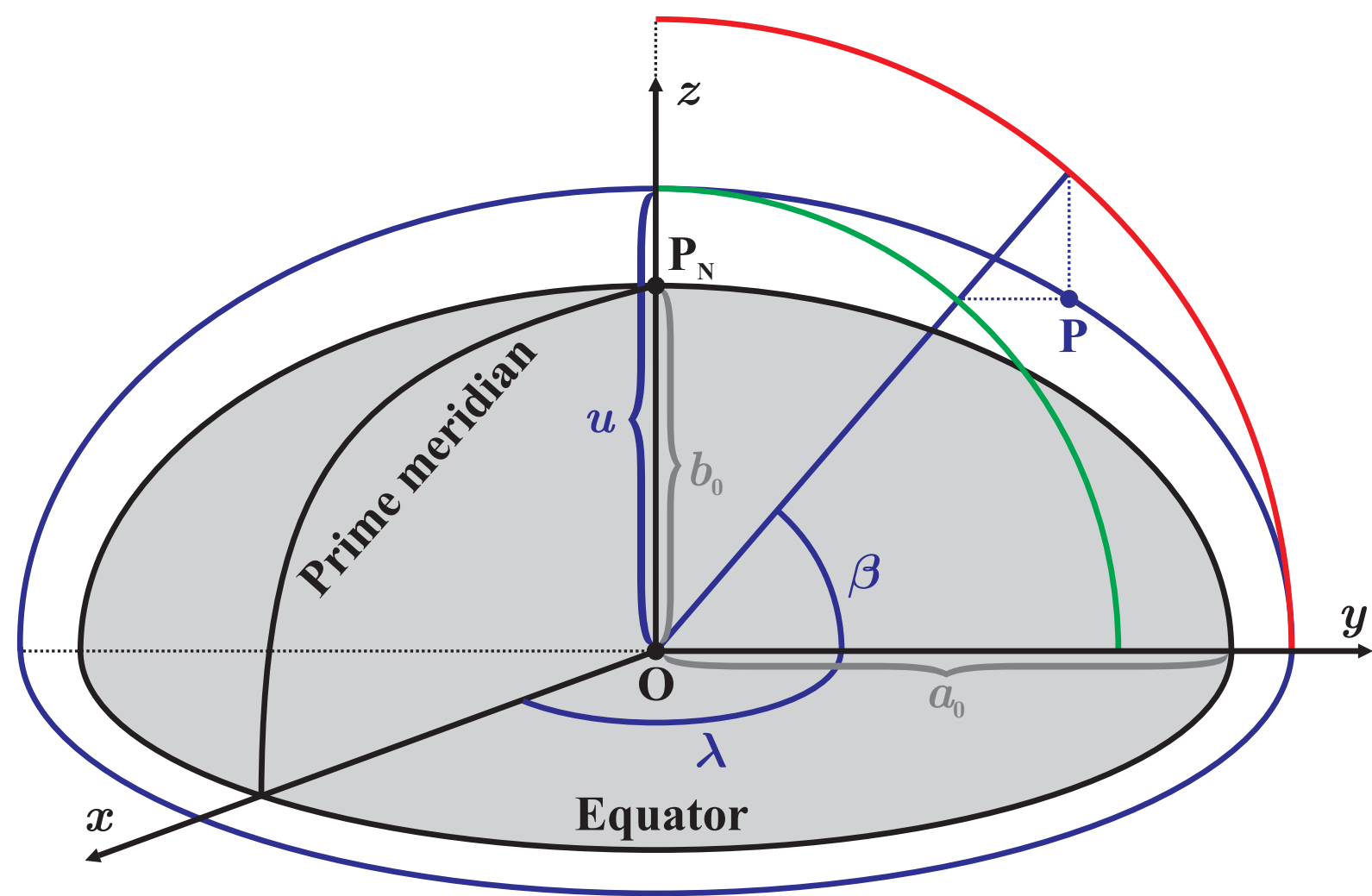

Figure 1: Graphical illustration of the planetocentric oblate spheroidal coordinates $u$ and $\Omega_{e}=(\beta, \lambda)$ at the point P. The grey-shaded oblate spheroidal surface represents the oblate reference spheroid with the semi-major axis $a_{0}$ and the semi-minor axis $b_{0}$. The oblate spheroidal surface bounded by the dark blue elliptical curves is the oblate confocal spheroid with the semi-major axis $\sqrt{u^{2}+\varepsilon^{2}}$ (being the radius of the red quarter circle) and the semi-minor axis $u$ (being the radius of the green quarter circle). $\mathrm{O}$ is the origin of the planetocentric Cartesian reference frame and $P_{N}$ is the North pole.

The spheroidal Newton integral transforms the bulk density $\rho$ and the geometry of the gravitating body (defined by integration limits) to the gravitational potential $V$ as follows (Moritz, 
1990, Sect. 5):

$V\left(u, \Omega_{e}\right)=G \int_{\Omega_{e}^{\prime}} \int_{u^{\prime}} \rho\left(u^{\prime}, \Omega_{e}^{\prime}\right) \mathcal{K}^{N}\left(u, \Omega_{e}, u^{\prime}, \Omega_{e}^{\prime}\right)\left(u^{\prime 2}+\varepsilon^{2} \sin ^{2} \beta^{\prime}\right) \mathrm{d} u^{\prime} \mathrm{d} \Omega_{e}^{\prime}$,

where $G$ is the universal gravitational constant, $\left(u, \Omega_{e}\right)$ refer to the position of the computation point and $\left(u^{\prime}, \Omega_{e}^{\prime}\right)$ specify the position of the integration element. The linear eccentricity $\varepsilon=$ $\sqrt{a_{0}^{2}-b_{0}^{2}}$ is related to the semi-major axis $a_{0}$ and the semi-minor axis $b_{0}$ of the oblate reference spheroid. The term $\left(u^{\prime 2}+\varepsilon^{2} \sin ^{2} \beta^{\prime}\right)$ under the integral originates from the metric coefficients of the planetocentric oblate spheroidal coordinate system (Thong and Grafarend, 1989). The explicit form of the oblate spheroidal surface element is $\mathrm{d} \Omega_{e}^{\prime}=\cos \beta^{\prime} \mathrm{d} \beta^{\prime} \mathrm{d} \lambda^{\prime}$.

The reciprocal distance $\mathcal{K}^{N}$ in Eq. (1), also known as Newton's kernel, is a function of the positions of both the computation point and the integration element. It is defined by (Neumann, 1848; Hobson, 1965, pp. 424-430):

$$
\begin{aligned}
& \mathcal{K}^{N}\left(u, \Omega_{e}, u^{\prime}, \Omega_{e}^{\prime}\right)=\left[\left(\sqrt{u^{2}+\varepsilon^{2}} \cos \beta \cos \lambda-\sqrt{u^{\prime 2}+\varepsilon^{2}} \cos \beta^{\prime} \cos \lambda^{\prime}\right)^{2}\right. \\
& \left.+\left(\sqrt{u^{2}+\varepsilon^{2}} \cos \beta \sin \lambda-\sqrt{u^{\prime 2}+\varepsilon^{2}} \cos \beta^{\prime} \sin \lambda^{\prime}\right)^{2}+\left(u \sin \beta-u^{\prime} \sin \beta^{\prime}\right)^{2}\right]^{-1 / 2} \\
& =\frac{i}{\varepsilon} \sum_{n=0}^{\infty} \sum_{m=-n}^{+n}(-1)^{|m|} \frac{(n-|m|) !}{(n+|m|) !} Q_{n,|m|}\left(i \frac{u}{\varepsilon}\right) P_{n,|m|}\left(i \frac{u^{\prime}}{\varepsilon}\right) \bar{Y}_{n, m}\left(\Omega_{e}\right) \bar{Y}_{n, m}\left(\Omega_{e}^{\prime}\right) .
\end{aligned}
$$

The expression after the first equality in Eq. (2) is the closed-form of Newton's kernel in terms of the planetocentric oblate spheroidal coordinates. The spectral form of Newton's kernel is given by the last expression of Eq. (2) and is uniformly convergent in the domain $u>u^{\prime}$. The symbol $i=\sqrt{-1}$ is the imaginary unit. The complex mathematical functions $P_{n,|m|}$ and $Q_{n,|m|}$ are respectively the un-normalised associated Legendre functions of the first and second kinds of the complex arguments of degree $n$ and order $m$. They determine the vertical variation of the Newton kernel. The horizontal variation of Newton's kernel is described by the scalar spheroidal harmonic (Abramowitz and Stegun, 1972, Sect. 8):

$\bar{Y}_{n, m}\left(\Omega_{e}\right)=\bar{P}_{n,|m|}(\sin \beta)\left\{\begin{array}{l}\cos m \lambda, \forall m \geq 0 \\ \sin |m| \lambda, \forall m<0\end{array}\right.$,

where $\bar{P}_{n,|m|}$ is the $4 \pi$ fully normalised associated Legendre function of the first kind for the real argument.

The spectral form of $\mathcal{K}^{N}$ allows for transformation of the Newton integral into a series representation. This is achieved by: 1) substituting the right-hand-side of Eq. (2) into Eq. (1); and 2) interchanging the order of summation and integration. This interchange is permitted with the assumption of the uniform convergence, which holds $\forall u>u^{\prime}$. The gravitational potential then reads:

$V\left(u, \Omega_{e}\right)=i \frac{G}{\varepsilon} \sum_{n=0}^{\infty} \sum_{m=-n}^{+n}(-1)^{|m|} \frac{(n-|m|) !}{(n+|m|) !} Q_{n,|m|}\left(i \frac{u}{\varepsilon}\right) \bar{Y}_{n, m}\left(\Omega_{e}\right)$ 
$\times \int_{\Omega_{e}^{\prime}} \int_{u^{\prime}} \rho\left(u^{\prime}, \Omega_{e}^{\prime}\right) \bar{Y}_{n, m}\left(\Omega_{e}^{\prime}\right) P_{n,|m|}\left(i \frac{u^{\prime}}{\varepsilon}\right)\left(u^{\prime 2}+\varepsilon^{2} \sin ^{2} \beta^{\prime}\right) \mathrm{d} u^{\prime} \mathrm{d} \Omega_{e}^{\prime}$.

The spheroidal harmonic spectrum is composed of the dimensionless fully-normalised spheroidal harmonic coefficients $\bar{C}_{n, m}^{e}$. These arise from the spheroidal harmonic expansion of the gravitational potential (Heiskanen and Moritz, 1967, Sect. 1-20):

$V\left(u, \Omega_{e}\right)=\frac{G M}{a} \sum_{n=0}^{\infty} \sum_{m=-n}^{+n} \frac{Q_{n,|m|}\left(i \frac{u}{\varepsilon}\right)}{Q_{n,|m|}\left(i \frac{b}{\varepsilon}\right)} \bar{C}_{n, m}^{e} \bar{Y}_{n, m}\left(\Omega_{e}\right)$

where $M$ is the mass of the gravitating body. This expansion is a solution of Laplace's equation $\nabla^{2} V=0$ for the planetocentric oblate spheroidal coordinate system and only holds true outside the gravitating masses. Equation (5) is absolutely convergent outside the oblate Brillouin spheroid (e.g., Hobson, 1965, p. 432). Such a spheroid is one that encloses all gravitating masses and is confocal to the oblate reference spheroid. Thus, the oblate Brillouin spheroid is geometrically defined by the semi-minor axis $b$ and the semi-major axis $a=\sqrt{b^{2}+\varepsilon^{2}}$.

Equations (4) and (5) are different representations of the same gravitational potential. By equating these two expressions and considering $u=b>u^{\prime}$, we obtain the desired formula for the forward-modelled spheroidal harmonic spectrum in the form:

$$
\begin{aligned}
& \bar{C}_{n, m}^{e}=i \frac{a}{M \varepsilon}(-1)^{|m|} \frac{(n-|m|) !}{(n+|m|) !} Q_{n,|m|}\left(i \frac{b}{\varepsilon}\right) \\
& \times \int_{\Omega_{e}^{\prime}} \int_{u^{\prime}} \rho\left(u^{\prime}, \Omega_{e}^{\prime}\right) \bar{Y}_{n, m}\left(\Omega_{e}^{\prime}\right) P_{n,|m|}\left(i \frac{u^{\prime}}{\varepsilon}\right)\left(u^{\prime 2}+\varepsilon^{2} \sin ^{2} \beta^{\prime}\right) \mathrm{d} u^{\prime} \mathrm{d} \Omega_{e}^{\prime} .
\end{aligned}
$$

Equation (6) is of importance when studying the physical meaning of the spheroidal harmonic coefficients (e.g., Grafarend and Ardalan, 1999). However, it is not suitable for numerical purposes, as it requires computing the factorials and the complex mathematical functions $P_{n,|m|}$ and $Q_{n,|m|}$. To avoid these practical limitations, Jekeli (1981, Eqs. (3.32) and (3.44)) introduced the following expressions for the un-normalised associated Legendre functions of the first and second kinds:

$P_{n,|m|}\left(i \frac{u^{\prime}}{\varepsilon}\right)=i^{n}\left(\frac{b}{\varepsilon}\right)^{n} \frac{(2 n) !}{2^{n} n !(n-|m|) !} p_{n,|m|}\left(\frac{u^{\prime}}{\varepsilon}\right)$,

$Q_{n,|m|}\left(i \frac{u}{\varepsilon}\right)=\frac{(-1)^{|m|}}{i^{n+1}}\left(\frac{\varepsilon}{b}\right)^{n+1} \frac{2^{n} n !(n+|m|) !}{(2 n+1) !} q_{n,|m|}\left(\frac{u}{\varepsilon}\right)$

where the symbols $p_{n,|m|}$ and $q_{n,|m|}$ stand for two real mathematical functions of the real arguments. These are referred to as the modified associated Legendre functions of the first and second kinds, respectively. By substituting Eqs. (7) and (8) into Eq. (6), we get an alternative formula for forward modelling to generate spheroidal harmonic coefficients:

$$
\begin{aligned}
& \bar{C}_{n, m}^{e}=\frac{a}{M b(2 n+1)} q_{n,|m|}\left(\frac{b}{\varepsilon}\right) \\
& \times \int_{\Omega_{e}^{\prime}} \int_{u^{\prime}} \rho\left(u^{\prime}, \Omega_{e}^{\prime}\right) \bar{Y}_{n, m}\left(\Omega_{e}^{\prime}\right) p_{n,|m|}\left(\frac{u^{\prime}}{\varepsilon}\right)\left(u^{\prime 2}+\varepsilon^{2} \sin ^{2} \beta^{\prime}\right) \mathrm{d} u^{\prime} \mathrm{d} \Omega_{e}^{\prime} .
\end{aligned}
$$


Equation (9) is discretised and numerically implemented in Sects. 3 and 4. This mathematical prescription is more general than the one used by (Wang and Yang, 2013), who restricted theirs to only horizontal variations of density. In addition, the simpler spherical harmonic spectrum (Heiskanen and Moritz, 1967, p. 59-60; Burša and Pěč, 1993, Sect.2-9) can be obtained from Eq. (9) by setting $\varepsilon \rightarrow 0$. In this degenerate case, $u^{\prime} \rightarrow r^{\prime}$ and $\beta^{\prime} \rightarrow \varphi^{\prime}$, where $r^{\prime}$ is the planetocentric spherical radius and $\varphi^{\prime}$ is the planetocentric spherical latitude. The oblate Brillioun spheroid becomes the Brillouin sphere of radius $a=b=R$, and the modified associated Legendre functions of the first and second kinds change to $q_{n,|m|}\left(\frac{b}{\varepsilon}\right) \rightarrow 1$ and $p_{n,|m|}\left(\frac{u^{\prime}}{\varepsilon}\right) \rightarrow\left(\frac{r^{\prime}}{R}\right)^{n}$. Thus, Eq. (9) also embeds the spherical spectral forward modelling as a degenerate case.

\section{Practical calculation of the spheroidal harmonic spectrum}

The geometry of the gravitating body and its bulk density distribution are commonly described by digital elevation models (DEMs) and digital density models (DDMs). To exploit these data for the computation of the spheroidal gravitational spectrum, the $3 \mathrm{D}$ volume integral in Eq. (9) has to be discretised.

The discretisation starts by dividing the volume of the gravitating body into what we term spheroidal tesseroids. We assign to every spheroidal tesseroid three indices $(\mu, \nu, \tau)$, and with the oblate spheroidal coordinates in the intervals $\beta^{\prime} \in\left[\beta_{\mu}^{\prime}, \beta_{\mu}^{\prime}+\Delta \beta^{\prime}\right], \lambda^{\prime} \in\left[\lambda_{\nu}^{\prime}, \lambda_{\nu}^{\prime}+\Delta \lambda^{\prime}\right]$, and $u^{\prime} \in\left[u_{\tau}^{\prime}, u_{\tau}^{\prime}+\Delta u_{\tau}^{\prime}\right]$. Thus, a spheroidal tesseroid is a $3 \mathrm{D}$ finite element bounded by two oblate confocal spheroids ( $u_{\tau}^{\prime}=$ const. and $u_{\tau}^{\prime}+\Delta u_{\tau}^{\prime}=$ const.), two one-sheet hyperboloids $\left(\beta_{\mu}^{\prime}=\right.$ const. and $\beta_{\mu}^{\prime}+\Delta \beta^{\prime}=$ const.), and two half-planes $\left(\lambda_{\nu}^{\prime}=\right.$ const. and $\lambda_{\nu}^{\prime}+\Delta \lambda^{\prime}=$ const.), see Fig. 2 . Such geometric interpretation is an extension of the spherical tesseroid formed by two concentric spheres, two half-cones, and two half-planes (Anderson, 1976, p. 48).

The discretisation steps $\Delta \beta^{\prime}$ and $\Delta \lambda^{\prime}$ are governed by the spatial resolutions of the DEMs and DDMs. The discretisation results in spheroidal gravitational spectra up to the maximum d/o $N_{\max }=180^{\circ} / \operatorname{MAX}\left(\Delta \beta^{\prime}, \Delta \lambda^{\prime}\right)$. The meaning of the discretisation step $\Delta u_{\tau}^{\prime}$ is twofold: 1$)$ $\Delta u_{\tau}^{\prime}=\Delta u^{\prime}\left(\beta^{\prime}, \lambda^{\prime}\right)$, i.e., it is a variable value determined by a DEM/DDM for all spheroidal tesseroids adjacent to the topographic surface of the gravitating body; 2) $\Delta u_{\tau}^{\prime}=\Delta u^{\prime}$, i.e., it is a fixed length for the other spheroidal tesseroids.

Each spheroidal tesseroid is specified by a bulk density represented by its mean value $\bar{\rho}\left(u_{\tau}^{\prime}, \beta_{\mu}^{\prime}, \lambda_{\nu}^{\prime}\right)$. Different mean density values can be assigned to each spheroidal tesseroid, thus permitting a full 3D implementation. Then, we can approximate the volume integral in Eq. (9) by the triple summation $\sum_{\mu, \nu, \tau}=\sum_{\mu} \sum_{\nu} \sum_{\tau}$ over all spheroidal tesseroids in 3D:

$$
\begin{aligned}
& \bar{C}_{n, m}^{e} \approx \frac{a}{M b(2 n+1)} q_{n,|m|}\left(\frac{b}{\varepsilon}\right) \sum_{\mu, \nu, \tau} \bar{\rho}\left(u_{\tau}^{\prime}, \beta_{\mu}^{\prime}, \lambda_{\nu}^{\prime}\right) \int_{\lambda_{\nu}^{\prime}}^{\lambda_{\nu}^{\prime}+\Delta \lambda^{\prime}}\left\{\begin{array}{c}
\cos m \lambda^{\prime} \\
\sin |m| \lambda^{\prime}
\end{array}\right\} \mathrm{d} \lambda^{\prime} \\
& \times\left[\int_{\beta_{\mu}^{\prime}}^{\beta_{\mu}^{\prime}+\Delta \beta^{\prime}} \bar{P}_{n,|m|}\left(\sin \beta^{\prime}\right) \cos \beta^{\prime} \mathrm{d} \beta^{\prime} \int_{u_{\tau}^{\prime}}^{u_{\tau}^{\prime}+\Delta u_{\tau}^{\prime}} p_{n,|m|}\left(\frac{u^{\prime}}{\varepsilon}\right) u^{\prime 2} \mathrm{~d} u^{\prime}\right. \\
& \left.+\varepsilon^{2} \int_{\beta_{\mu}^{\prime}}^{\beta_{\mu}^{\prime}+\Delta \beta^{\prime}} \bar{P}_{n,|m|}\left(\sin \beta^{\prime}\right) \sin ^{2} \beta^{\prime} \cos \beta^{\prime} \mathrm{d} \beta^{\prime} \int_{u_{\tau}^{\prime}}^{u_{\tau}^{\prime}+\Delta u_{\tau}^{\prime}} p_{n,|m|}\left(\frac{u^{\prime}}{\varepsilon}\right) \mathrm{d} u^{\prime}\right], \begin{array}{l}
\forall m \geq 0 \\
\forall m<0
\end{array} .
\end{aligned}
$$

To employ the discretised volume integral in practical calculations, we provided formulas for the modified associated Legendre function of the second kind $q_{n,|m|}$ and solved the five integrals over the oblate spheroidal coordinates $u^{\prime}, \beta^{\prime}$, and $\lambda^{\prime}$. 


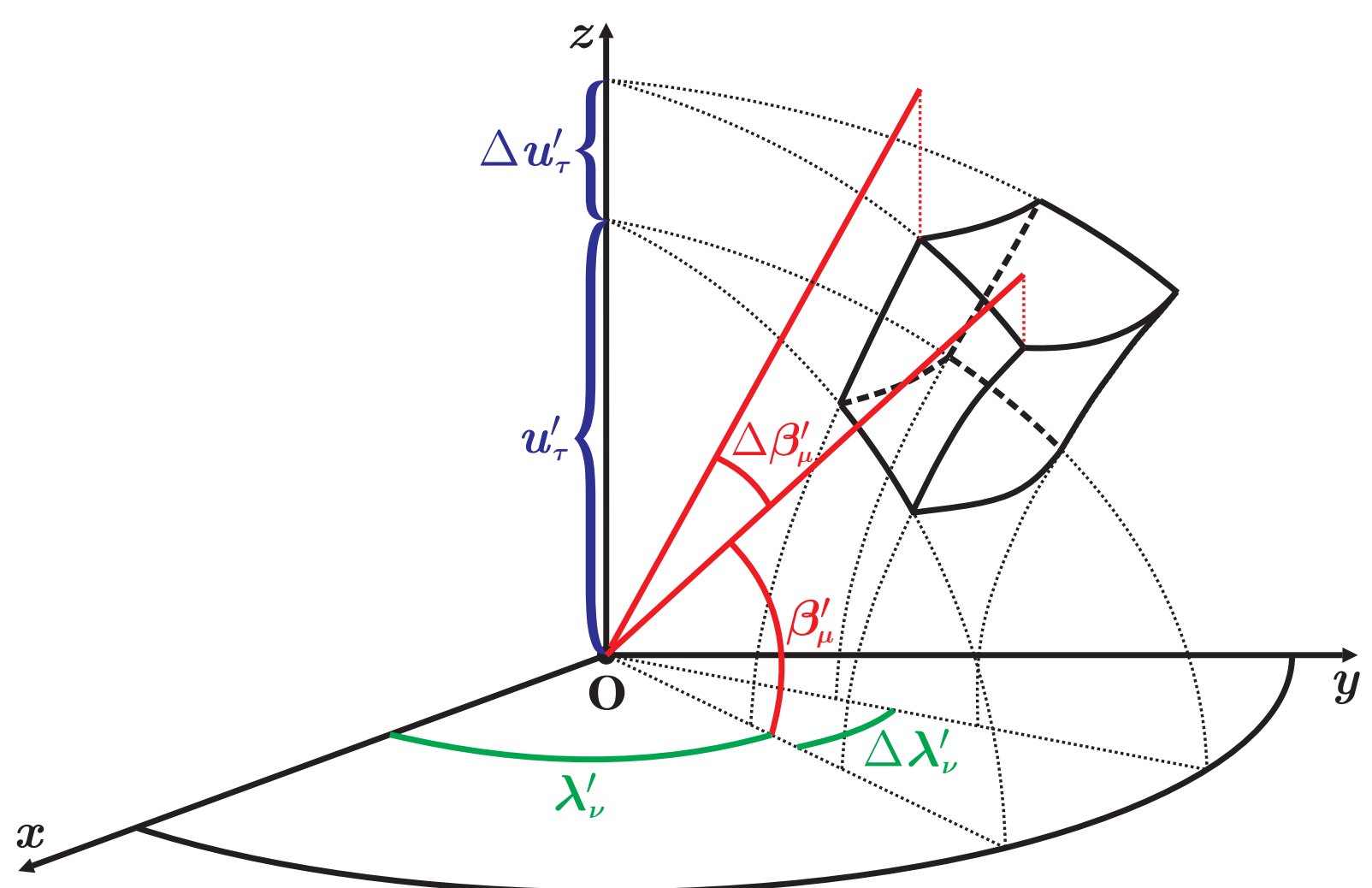

Figure 2: Graphical illustration of a spheroidal tesseroid. The red half-lines are asymptotes of the hyperbolas defining all points with $\beta_{\mu}^{\prime}=$ const. or $\beta_{\mu}^{\prime}+\Delta \beta^{\prime}=$ const. in the half-plane $\lambda_{\nu}^{\prime}$.

\subsection{Efficiency gains through recursions}

The function $q_{n,|m|}$ can be computed efficiently by recursive formulas. To avoid possible numerical instabilities, an operational approach that combines forward and backward recursions was suggested by (Gil and Segura, 1988). Alternatively, one may exploit the hypergeometric representation of $q_{n,|m|}$ of the form (Hobson, 1965, p. 108):

$q_{n,|m|}\left(\frac{u}{\varepsilon}\right)=\left(\frac{b}{\sqrt{u^{2}+\varepsilon^{2}}}\right)^{n+1}{ }_{2} F_{1}\left(\frac{n+|m|+1}{2}, \frac{n-|m|+1}{2}, \frac{2 n+3}{2}, \frac{\varepsilon^{2}}{u^{2}+\varepsilon^{2}}\right)$.

We prefer to use Eq. (11) as it preserves the numerical accuracy of $q_{n,|m|}$ for arbitrary d/o. In this article, the Gauss hypergeometric function ${ }_{2} F_{1}$ was calculated by the routine gsl_sf_hyperg_2F1, one of the mathematical functions of the GNU Scientific Library (Galassi et al., 2013).

The integrals over the longitude $\lambda^{\prime}$ are the simplest, as we can find exactly:

$$
\int_{\lambda_{\nu}^{\prime}}^{\lambda_{\nu}^{\prime}+\Delta \lambda^{\prime}}\left\{\begin{array}{c}
\cos m \lambda^{\prime} \\
\sin |m| \lambda^{\prime}
\end{array}\right\} \mathrm{d} \lambda^{\prime}=\left\{\begin{array}{l}
A_{m} \cos \left(m \nu \Delta \lambda^{\prime}\right)+B_{m} \sin \left(m \nu \Delta \lambda^{\prime}\right), \forall m \geq 0 \\
A_{|m|} \sin \left(|m| \nu \Delta \lambda^{\prime}\right)-B_{|m|} \cos \left(|m| \nu \Delta \lambda^{\prime}\right), \forall m<0
\end{array} .\right.
$$

Equations (10) and (12) were obtained by assigning $\lambda_{\nu}^{\prime}=\nu \Delta \lambda^{\prime}$, which is valid for regularly sampled data. The ancillary coefficients $A_{m}$ and $B_{m}$ read:

$$
A_{m}=\left\{\begin{array}{ll}
\Delta \lambda^{\prime}, & m=0 \\
\frac{\sin \left(m \Delta \lambda^{\prime}\right)}{m}, & \forall m \geq 1
\end{array} \quad, \quad B_{m}=\left\{\begin{array}{ll}
0, & m=0 \\
\frac{\cos \left(m \Delta \lambda^{\prime}\right)-1}{m}, & \forall m \geq 1
\end{array} .\right.\right.
$$


The two integrals of $\bar{P}_{n,|m|}$ in Eq. (10) are more complicated. Their analytical expressions can be derived for low ds/os. However, this is not feasible when $N_{\max }$ reaches several hundreds or thousands. Recursions are therefore used in our practical calculations, see the Supplementary Material. To calculate these integrals up to ultra-high ds/os without numerical problems, we used the computational procedure based on the X-number formulation (e.g., Fukushima, 2012, 2014). We abbreviate the two integrals as:

$\int_{\beta_{\mu}^{\prime}}^{\beta_{\mu}^{\prime}+\Delta \beta^{\prime}} \bar{P}_{n,|m|}\left(\sin \beta^{\prime}\right) \cos \beta^{\prime} \mathrm{d} \beta^{\prime}=\overline{I P}_{n,|m|}^{\mu}$,

$\int_{\beta_{\mu}^{\prime}}^{\beta_{\mu}^{\prime}+\Delta \beta^{\prime}} \bar{P}_{n,|m|}\left(\sin \beta^{\prime}\right) \sin ^{2} \beta^{\prime} \cos \beta^{\prime} \mathrm{d} \beta^{\prime}=\overline{I P S}_{n,|m|}^{\mu}$.

Similarly, the two integrals of $p_{n,|m|}$ in Eq. (10) are calculated by recursive formulas. These integrals are defined by the shorthand notation:

$\int_{u_{\tau}^{\prime}}^{u_{\tau}^{\prime}+\Delta u_{\tau}^{\prime}} p_{n,|m|}\left(\frac{u^{\prime}}{\varepsilon}\right) \mathrm{d} u^{\prime}=\overline{i p}_{n,|m|}^{\tau}$

$\int_{u_{\tau}^{\prime}}^{u_{\tau}^{\prime}+\Delta u_{\tau}^{\prime}} p_{n,|m|}\left(\frac{u^{\prime}}{\varepsilon}\right) u^{\prime 2} \mathrm{~d} u^{\prime}=\overline{i p u}_{n,|m|}^{\tau}$

To the best of our knowledge, recursions for $\overline{i p}_{n,|m|}^{\tau}$ and $\overline{i p u}_{n,|m|}^{\tau}$ have not been presented before. We derive these recursion formulas and investigate their numerical behaviour in the Supplementary Material.

Equations (12) and (14)-(17) allow the discretised volume integral of Eq. (10) to be written more compactly as:

$\bar{C}_{n, m}^{e}=\frac{a}{M b(2 n+1)} q_{n,|m|}\left(\frac{b}{\varepsilon}\right)$

$\times \sum_{\mu, \nu, \tau} \bar{\rho}\left(u_{\tau}^{\prime}, \beta_{\mu}^{\prime}, \lambda_{\nu}^{\prime}\right)\left\{\begin{array}{l}A_{m} \cos \left(m \nu \Delta \lambda^{\prime}\right)+B_{m} \sin \left(m \nu \Delta \lambda^{\prime}\right) \\ A_{|m|} \sin \left(|m| \nu \Delta \lambda^{\prime}\right)-B_{|m|} \cos \left(|m| \nu \Delta \lambda^{\prime}\right)\end{array}\right\}$

$\times\left[\overline{I P}_{n,|m|}^{\mu} \overline{i p u}_{n,|m|}^{\tau}+\varepsilon^{2} \overline{I P S}_{n,|m|}^{\mu} \overline{i p}_{n,|m|}^{\tau}\right], \quad \begin{array}{r}\forall m \geq 0 \\ \forall m<0\end{array}$

This expression can be exploited for numerical evaluation. However, summations over the three indices $(\mu, \nu, \tau)$ may be computationally challenging, particularly for small discretisation steps.

\subsection{Efficiency gains through Fourier transform}

We can speed up the numerical evaluation further by introducing the Fourier coefficients:

$$
\left.\begin{array}{l}
D_{n, m}^{\mu} \\
E_{n, m}^{\mu}
\end{array}\right\}=\sum_{\nu}\left[\sum_{\tau} \bar{\rho}\left(u_{\tau}^{\prime}, \beta_{\mu}^{\prime}, \lambda_{\nu}^{\prime}\right) \overline{i p}_{n, m}^{\tau}\right]\left\{\begin{array}{l}
\cos \left(m \nu \Delta \lambda^{\prime}\right) \\
\sin \left(m \nu \Delta \lambda^{\prime}\right)
\end{array}\right.
$$


$=\left\{\begin{array}{l}\Re\left\{\mathcal{F}\left[\sum_{\tau} \bar{\rho}\left(u_{\tau}^{\prime}, \beta_{\mu}^{\prime}, \lambda_{\nu}^{\prime}\right) \overline{i p}_{n, m}^{\tau}\right]\right\} \\ \Im\left\{\mathcal{F}\left[\sum_{\tau} \bar{\rho}\left(u_{\tau}^{\prime}, \beta_{\mu}^{\prime}, \lambda_{\nu}^{\prime}\right) \overline{i p}_{n, m}^{\tau}\right]\right\}\end{array}, \forall m \geq 0\right.$.

The two summations over the indices $\nu$ and $\tau$ have been transferred into the real part $\Re$ and imaginary part $\Im$ of the Fourier image $\mathcal{F}$ of $\sum_{\tau} \bar{\rho}\left(u_{\tau}^{\prime}, \beta_{\mu}^{\prime}, \lambda_{\nu}^{\prime}\right) \overline{i p}_{n, m}^{\tau}$. Computational acceleration comes from the fact that the right-hand-side of Eq. (19) can efficiently be computed by using 1D fast/discrete Fourier transform algorithms (Cooley and Tukey, 1965). In a similar way, we define the Fourier coefficients corresponding to the integrals $\overline{i p u}_{n,|m|}^{\tau}$, i.e.:

$$
\begin{aligned}
& \left.\begin{array}{l}
D U_{n, m}^{\mu} \\
E U_{n, m}^{\mu}
\end{array}\right\}=\sum_{\nu}\left[\sum_{\tau} \bar{\rho}\left(u_{\tau}^{\prime}, \beta_{\mu}^{\prime}, \lambda_{\nu}^{\prime}\right) \overline{i p u}_{n, m}^{\tau}\right]\left\{\begin{array}{l}
\cos \left(m \nu \Delta \lambda^{\prime}\right) \\
\sin \left(m \nu \Delta \lambda^{\prime}\right)
\end{array}\right. \\
& =\left\{\begin{array}{l}
\Re\left\{\mathcal{F}\left[\sum_{\tau} \bar{\rho}\left(u_{\tau}^{\prime}, \beta_{\mu}^{\prime}, \lambda_{\nu}^{\prime}\right) \overline{i p u}_{n, m}^{\tau}\right]\right\} \\
\Im\left\{\mathcal{F}\left[\sum_{\tau} \bar{\rho}\left(u_{\tau}^{\prime}, \beta_{\mu}^{\prime}, \lambda_{\nu}^{\prime}\right) \overline{i p u}_{n, m}^{\tau}\right]\right\}
\end{array}, \forall m \geq 0 .\right.
\end{aligned}
$$

Making use of the Fourier coefficients of Eqs. (19) and (20), the efficient discretised volume integral for calculating the spheroidal harmonic spectrum reads:

$$
\begin{aligned}
& \bar{C}_{n, m}^{e}=\frac{a}{M b(2 n+1)} q_{n,|m|}\left(\frac{b}{\varepsilon}\right) \sum_{\mu}\left[\overline{I P}_{n,|m|}^{\mu}\left\{\begin{array}{l}
A_{m} D U_{n, m}^{\mu}+B_{m} E U_{n, m}^{\mu} \\
A_{|m|} E U_{n,|m|}^{\mu}-B_{|m|} D U_{n,|m|}^{\mu}
\end{array}\right\}\right. \\
& \left.+\varepsilon^{2} \overline{I P S}_{n,|m|}^{\mu}\left\{\begin{array}{l}
A_{m} D_{n, m}^{\mu}+B_{m} E_{n, m}^{\mu} \\
A_{|m|} E_{n,|m|}^{\mu}-B_{|m|} D_{n,|m|}^{\mu}
\end{array}\right\}\right], \begin{array}{l}
\forall m \geq 0 \\
\forall m<0
\end{array} .
\end{aligned}
$$

An equivalent application of the 1D fast/discrete Fourier transform was proposed for forward modelling of spherical harmonic coefficients (Šprlák et al., 2018). It is computationally less expensive than that presented here, however, because the corresponding Fourier coefficients depend only on the spherical harmonic degree $n$ and the longitude index $\mu$.

We emphasise that the key aspect of the discretisation is the explicit separation into the five integrals, see Eq. (10). Each of these integrals includes mathematical functions of only one variable, i.e., $u^{\prime}, \beta^{\prime}$, or $\lambda^{\prime}$, and allows for recursions. In contrast, Wang and Yang (2013) did not separate the integrals over the individual variables. Namely, they considered the variable $\beta^{\prime}$ in the integrals over $u^{\prime}$, see (Wang and Yang, 2013, Eq. (32)) and suggested using Laurent series for practical calculation of these integrals. However, in comparison to recursions, accurate computation of the Laurent series includes an extra loop and many series terms may be required for ultra-high ds/os. Thus, the formulation by (Wang and Yang, 2013) is computationally more demanding than our explicit formalism with simple recursions.

\section{Numerical experiments}

The purpose of this Section is twofold. Firstly, we test the accuracy of our forward modelling for the synthetic gravitational field of a homogeneous spheroidal shell. Secondly, we demonstrate suitability of the spheroidal modelling with respect to its simpler spherical counterpart for gravitational field generated by a highly eccentric planetary body. We also consider the Earth's Moon as the degenerate spherical case. 
We have developed a computer program in C. It implements the discretised volume integral for calculating the spheroidal harmonic coefficients, see Sect. 3, and the recursions from the Supplementary Material. A manual for the computer program can also be found in the Supplementary Material. The program requires a DEM that is composed of regularly sampled spheroidal heights above an oblate reference spheroid $u^{\prime}\left(\beta^{\prime}, \lambda^{\prime}\right)-b_{0}$. This is different from Wang and Yang (2013), who approximated the spheroidal heights $u^{\prime}\left(\beta^{\prime}, \lambda^{\prime}\right)-b_{0}$ by the geodetic (ellipsoidal) heights $h$ in the geodetic (Gauss ellipsoidal) coordinate system (Grafarend and Engels, 1992).

Among other candidates, we only considered the planetary bodies 1 Ceres and the Moon, which are different in size and shape, see Table 1. In particular, 1 Ceres is significantly flattened $(f \approx 0.0747)$ towards the poles, whereas the Moon is nearly spherical (with flattening $f \approx$ 0.0003). This choice is intentional, because it allows for identification of potential challenges or benefits of the spheroidal forward modelling for different amounts of flattening. Also, detailed DEMs of these bodies are available (Preusker et al., 2016; Smith et al., 2010), which makes them suitable for testing high-resolution spheroidal forward modelling.

\begin{tabular}{|c|c|c|c|}
\hline Planetary body & Parameter & Value & Reference \\
\hline \multirow{11}{*}{1 Ceres } & $a_{0}$ & $482000 \mathrm{~m}$ & (Preusker et al., 2016) \\
\hline & $b_{0}$ & $446000 \mathrm{~m}$ & (Preusker et al., 2016) \\
\hline & $f$ & 0.0746887966804979 & - \\
\hline & $a$ & $490784.18825487848 \mathrm{~m}$ & - \\
\hline & $b$ & $455479 \mathrm{~m}$ & - \\
\hline & $R_{0}$ & $470000 \mathrm{~m}$ & (Konopliv et al., 2018) \\
\hline & $R$ & $487057 \mathrm{~m}$ & - \\
\hline & $R_{C}$ & $437000 \mathrm{~m}$ & (Konopliv et al., 2018) \\
\hline & $\rho$ & $1400 \mathrm{~kg} \mathrm{~m}^{-3}$ & (Konopliv et al., 2018) \\
\hline & $G M$ & $6.26273587422 \times 10^{10} \mathrm{~m}^{3} \mathrm{~s}^{-2}$ & (Konopliv et al., 2018) \\
\hline & $\operatorname{MAX}\left[u^{\prime}\left(\beta^{\prime}, \lambda^{\prime}\right)-b_{0}\right]$ & $9478.267 \mathrm{~m}$ & (Preusker et al., 2016) \\
\hline \multirow{11}{*}{ Moon } & $a_{0}$ & $1737325 \mathrm{~m}$ & (Ardalan and Karimi, 2014) \\
\hline & $b_{0}$ & $1736789 \mathrm{~m}$ & (Ardalan and Karimi, 2014) \\
\hline & $f$ & 0.0003085202826184 & - \\
\hline & $a$ & $1748182.66997588123 \mathrm{~m}$ & - \\
\hline & $b$ & $1747650 \mathrm{~m}$ & - \\
\hline & $R_{0}$ & $1737400 \mathrm{~m}$ & (Smith et al., 2010) \\
\hline & $R$ & $1748200 \mathrm{~m}$ & - \\
\hline & $R_{C}$ & $1703400 \mathrm{~m}$ & (Wieczorek et al., 2013) \\
\hline & $\rho$ & $2550 \mathrm{~kg} \mathrm{~m}^{-3}$ & (Wieczorek et al., 2013) \\
\hline & $G M$ & $4.9028003055554 \times 10^{12} \mathrm{~m}^{3} \mathrm{~s}^{-2}$ & (Konopliv et al., 2013) \\
\hline & $\operatorname{MAX}\left[u^{\prime}\left(\beta^{\prime}, \lambda^{\prime}\right)-b_{0}\right]$ & $10855.977 \mathrm{~m}$ & (Smith et al., 2010) \\
\hline
\end{tabular}

Table 1: Summary of the parameters used in the numerical experiments. 
Due to the lack of DDMs, we simply assumed a constant bulk density $\rho=\rho\left(u^{\prime}, \Omega_{e}^{\prime}\right)=$ const. for all spheroidal tesseroids modelled by the DEM. However, the mathematical framework presented herein does allow for implementation of a 3D DDM. Values were chosen for 1 Ceres and the Moon based on the available estimates of the mean crustal densities (Wieczorek et al., 2013; Konopliv et al., 2018), see Table 1. We also note that the spheroidal coordinate $u^{\prime}$ was discretised by only one step, the size of which equals the spheroidal height of the volume integration element, i.e., $\Delta u_{\tau}^{\prime}=\Delta u^{\prime}\left(\beta^{\prime}, \lambda^{\prime}\right)=u^{\prime}\left(\beta^{\prime}, \lambda^{\prime}\right)-b_{0}$. We emphasise that our final sets of computed spheroidal harmonic coefficients always refer to each respective oblate Brillouin spheroid, see Table 1.

\subsection{Gravitational field of a homogeneous spheroidal shell}

We begin our numerical experiments with the gravitational field of a homogeneous spheroidal shell for which there is a closed-form analytical solution. The true spheroidal harmonic coefficients of the homogeneous spheroidal shell (assigned also with the superscript $t$ ) are zero except for (e.g., Wang, 1988; Pohánka, 2011):

$\bar{C}_{0,0}^{e, t}=\frac{4 \pi \rho a}{3 \varepsilon M}\left[u_{2}^{\prime 3}-u_{1}^{\prime 3}+\varepsilon^{2}\left(u_{2}^{\prime}-u_{1}^{\prime}\right)\right] \arctan \frac{\varepsilon}{b}$,

$\bar{C}_{2,0}^{e, t}=\frac{2 \pi \rho \varepsilon a}{3 \sqrt{5} M}\left[u_{2}^{\prime}\left(1+\frac{u_{2}^{\prime 2}}{\varepsilon^{2}}\right)-u_{1}^{\prime}\left(1+\frac{u_{1}^{\prime 2}}{\varepsilon^{2}}\right)\right]\left[\left(1+\frac{3 b^{2}}{\varepsilon^{2}}\right) \arctan \frac{\varepsilon}{b}-\frac{3 b}{\varepsilon}\right]$.

The analytical expressions in Eqs. (22a) and (22b) show that $\bar{C}_{0,0}^{e, t}$ and $\bar{C}_{2,0}^{e, t}$ are given by the parameters $a_{0}, b_{0}, a, b, \rho, M, u_{1}^{\prime}$, and $u_{2}^{\prime}$. Numerical values of the first five parameters are listed in Table 1. The semi-major and semi-minor axes $a_{0}$ and $b_{0}$ define the best-fitting oblate spheroids (Ardalan and Karimi, 2014; Preusker et al., 2016).

The semi-minor axes $b$ of the oblate Brillouin spheroids were obtained by finding the maxima of the spheroidal coordinate $u^{\prime}\left(\beta^{\prime}, \lambda^{\prime}\right)$ from the DEMs. The maxima were rounded up to the nearest metre for 1 Ceres and to the nearest 50 metres for the Moon. This guarantees that all gravitating masses are enclosed within their oblate Brillouin spheroids. The semi-major axes $a$ were then calculated from the semi-minor axes $b$ and the linear eccentricities $\varepsilon$.

Table 1 also provides numerical values of the planetocentric products $G M$. These originate from the global gravitational field models (Konopliv et al., 2013, 2018). The masses $M$ of the two planetary bodies, as required by Eqs. (22a) and (22b), were obtained by dividing $G M$ by the universal gravitational constant $G=6.6742 \times 10^{-11} \mathrm{~kg}^{-1} \mathrm{~m}^{3} \mathrm{~s}^{-2}$ (Mohr and Taylor, 2005).

The lower spheroidal surfaces of the homogeneous spheroidal shells are defined by the semiminor axes $u_{1}^{\prime}$. We selected these surfaces such that $u_{1}^{\prime}=b_{0}$, i.e., they coincide with the oblate reference spheroids. The upper spheroidal surfaces are given by the semi-minor axes $u_{2}^{\prime}=b_{0}+\operatorname{MAX}\left[u^{\prime}\left(\beta^{\prime}, \lambda^{\prime}\right)-b_{0}\right]$. These choices of the lower and the upper spheroidal surfaces always guaranteed that $u_{1}^{\prime} \leq u_{2}^{\prime}<b$ so that the series is uniformly convergent. This is in agreement with the theoretical requirements in Sects. 2 and 3.

We computed spheroidal harmonic coefficients for the spheroidal shells by our forward modelling software to verify its accuracy. To be consistent with the spheroidal gravitational spectrum from the solutions of the analytical expressions in Eqs. (22a) and (22b), all parameters were set to identical values. The DEM was represented by a constant grid of spheroidal heights $\operatorname{MAX}\left[u^{\prime}\left(\beta^{\prime}, \lambda^{\prime}\right)-b_{0}\right]$, see Table 1 . Synthetic grids of the constant spheroidal heights were discretised with the steps $\Delta \beta^{\prime}=\Delta \lambda^{\prime}=1 / 14^{\circ}$. This corresponds to the maximum d/o $N_{\max }=2519$ in the spectral domain. 
The finest-resolution global gravitational field models of 1 Ceres and the Moon were determined, respectively, up to the maximum ds/os $N_{\max }=18$ (Konopliv et al., 2018) and 1500 (Konopliv et al., 2014). The spectral resolution of the forward-modelled spheroidal harmonic spectra considered in this study is much higher. It was chosen deliberately to examine the performance of the spheroidal forward modelling for ultra-high harmonic expansions. This was also allowed by the spatial resolutions of the available DEMs.

The highest spectral resolution has to be set reasonably, because the spheroidal approach may be computationally challenging for as high as $N_{\max }=2519$. Our high-resolution modelling was performed at the High Performance Computing Facilities of the University of Newcastle. We divided the corresponding synthetic grids of the constant spheroidal heights into 90 bands in the direction of the spheroidal latitude $\beta^{\prime}$. Subsequently, computations were carried out and completed in approximately less than two days by exploiting 90 processors simultaneously, i.e., one processor for each latitudinal band. Our further numerical experiments showed that the spheroidal forward modelling up to $N_{\max }=359$ took only few hours by using an ordinary desktop computer (equipped with $32 \mathrm{~GB}$ of RAM, eight processors of frequency $3.4 \mathrm{GHz}$, while exploiting only one processor at a runtime, and with a Linux 64-bit operating system).

To investigate the numerical performance of the spheroidal approach, we firstly determined the relative accuracy of the coefficients $\bar{C}_{0,0}^{e}$ and $\bar{C}_{2,0}^{e}$ by the formula:

$\delta \bar{C}_{i, 0}^{e}=\frac{\left|\bar{C}_{i, 0}^{e, t}-\bar{C}_{i, 0}^{e}\right|}{\bar{C}_{i, 0}^{e, t}}, \quad i=1,2$.

The superscript $t$ indicates the "true" synthetic coefficients computed by Eqs. (22a) and (22b), while the coefficients without the superscript $t$ were calculated by our forward modelling. The relative accuracy is $\delta \bar{C}_{0,0}^{e} \approx 7.4 \times 10^{-14}$ and $\delta \bar{C}_{2,0}^{e} \approx 8.0 \times 10^{-14}$ for 1 Ceres, approaching numerical error of double precision computation. Almost identical relative accuracy being $\delta \bar{C}_{0,0}^{e} \approx 6.3 \times 10^{-14}$ and $\delta \bar{C}_{2,0}^{e} \approx 6.1 \times 10^{-14}$ was found for the Moon.

We also examined the square root of degree variances, which can be defined for an oblate spheroidal surface given by the coordinate $u$ and confocal with the oblate reference spheroid as follows:

$\sigma_{n}^{e}(u)=\frac{\sqrt{u^{2}+\varepsilon^{2}}}{a} \sqrt{\sum_{m=-n}^{+n}\left[\frac{Q_{n,|m|}\left(i \frac{u}{\varepsilon}\right)}{Q_{n,|m|}\left(i \frac{b}{\varepsilon}\right)} \bar{C}_{n, m}^{e}\right]^{2}}$.

The rationale for using the degree variances of a spheroidal harmonic spectrum is explained in (Lowes and Winch, 2012).

The power spectrum $\sigma_{n}^{e}(b)$ of Eq. (24) should theoretically be zero for the homogeneous spheroidal shells $(\forall n \neq 0,2)$, see Fig. 3. The accuracy reaches the lowest values, i.e., highest square root of degree variances, of $\sim 10^{-18}$ for low $\mathrm{d} / \mathrm{o}$. Above $\mathrm{d} / \mathrm{o} \sim 600$, the square roots of degree variances are consistently below $\sim 10^{-20}$. This degree-dependence may be explained by the decreasing magnitudes of the associated Legendre functions of the first kind. We postulate that the slightly discontinuous behaviour of the two curves in Fig. 3 originates from the Xnumber algorithm by (Fukushima, 2012, 2014). However, these are numerically negligible and do not need any further consideration. Similar numerical performance has been reported for the spherical forward modelling (Šprlák et al., 2018), but their "true" analytical solution was instead inferred by a homogeneous spherical shell.

This numerical experiment with the homogeneous spheroidal shells infers successful implementation of our algorithm for the spheroidal forward modelling. We simultaneously showed 


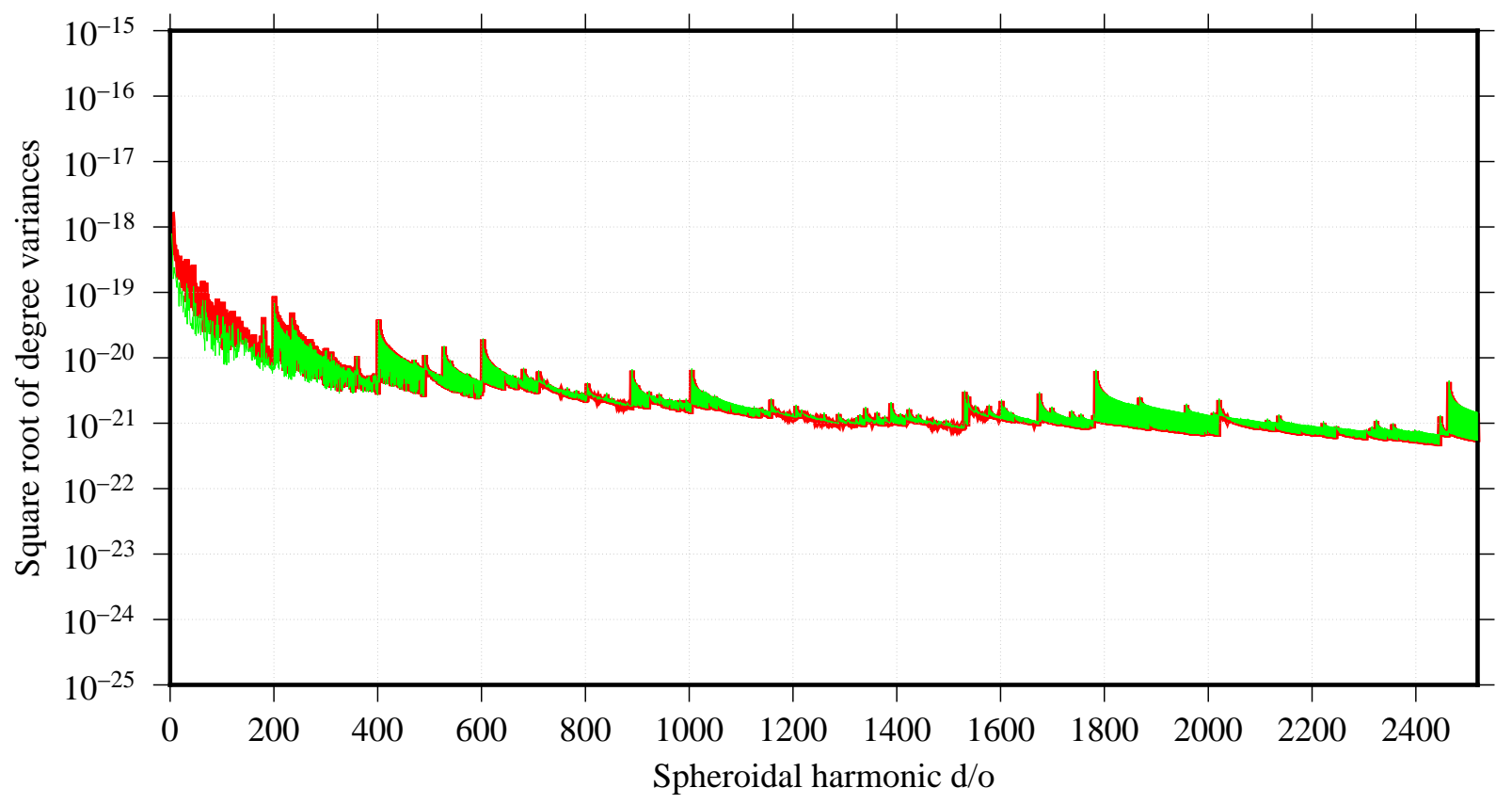

Figure 3: Square root of degree variances from the spheroidal harmonic spectra as calculated by the spheroidal forward modelling for 1 Ceres (red) and the Moon (green). The spheroidal harmonic spectra were computed at the oblate Brillouin spheroids, i.e., $u=b$, and inferred by the homogeneous spheroidal shells for each body.

that the spheroidal approach is computationally feasible and accurate even up to d/o 2519 for the nearly spherical Moon and for the significantly flattened 1 Ceres.

\subsection{Gravitational field from a homogeneous crust}

We now proceed to compute realistic gravitational fields inferred by the assumed-homogeneous crusts of 1 Ceres and the Moon. These could be applied for geophysical investigations or spacecraft navigation purposes (e.g., Moritz, 1990; Miller, 2019). However, our primary aim is to demonstrate the benefit of the spheroidal forward modelling in comparison to its simpler spherical analogue by (Šprlák et al., 2018) for application to highly elliptical bodies.

The numerical experiments were performed similarly to the synthetic one (Sect. 4.1). Thus, the majority of parameters were preserved. To calculate the global gravitational fields inferred by the topographic masses of 1 Ceres and of the Moon, only two aspects were changed.

First, both the spherical and the spheroidal forward modelling were performed using DEMs only, as DDMs are not yet available. The available DEMs of 1 Ceres and of the Moon are discretised with steps $\Delta \beta^{\prime}=\Delta \lambda^{\prime}=1 / 60^{\circ}$ and $1 / 64^{\circ}$, respectively. The original DEMs were smoothed to the spatial resolution of $1 / 14^{\circ}$, which corresponds to $N_{\max }=2519$. The smoothing was carried out by calculating the blockmean values of the spherical and of the spheroidal heights using blockmean from the Generic Mapping Tools (Wessel et al., 2013). The spherical heights are defined as $r^{\prime}\left(\varphi^{\prime}, \lambda^{\prime}\right)-R_{0}$, where $R_{0}$ is the radius of the reference sphere. Numerical values of $R_{0}$ for 1 Ceres and for the Moon (Smith et al., 2010; Konopliv et al., 2018) are listed in Table 1.

Second, topographic masses were bounded at the base of the spherical radius $R_{C}$. Values of $R_{C}$ are different for the two planetary bodies, see Table 1 . These originate from the estimates of the average crustal thickness (Wieczorek et al., 2013; Konopliv et al., 2018), which can be calculated as $R_{0}-R_{C}$. Thus, theoretically, identical topographic masses were considered for 
both forward modelling techniques. Practically, small differences may exist and originate from the discretisation.

Application of the spherical forward modelling results in spherical harmonic spectra referred to the Brillouin sphere. We determined the radii of the Brillouin spheres $R$ by: 1) finding the maxima of the spherical radius $r^{\prime}\left(\varphi^{\prime}, \lambda^{\prime}\right)$, and 2) rounding up to the nearest one metre for 1 Ceres and up to the nearest 50 meters for the Moon, see Table 1. This is in correspondence to determining the semi-minor axes $b$ of the oblate Brillouin spheroids, see above.

The parameters $a, b$, and $R$ in Table 1 show the geometric relationship between the Brillouin surfaces. The deviations between the oblate Brillouin spheroid of 1 Ceres and its spherical equivalent reach $\sim+4.7 \mathrm{~km}$ at the equator and $\sim-31.6 \mathrm{~km}$ at the poles. The positive and negative signs indicate whether the oblate Brillouin spheroid is located above or below the Brillouin sphere. For the Moon, the Brillouin sphere completely envelopes the oblate Brillouin spheroid. These two lunar Brillouin surfaces differ by $\sim-18 \mathrm{~m}$ at the equator and $\sim-550 \mathrm{~m}$ at the poles.

The oblate Brillouin spheroids of 1 Ceres and of the Moon have smaller volumes than the corresponding Brillouin spheres. This indicates a larger convergence space above the topography and, eventually, preference of the spheroidal modelling, especially for highly elliptical bodies. Below, we quantified performance of the spherical and the spheroidal forward modelling by examining convergence/divergence properties of their power spectra. We selected the power spectra as metric because they are a useful tool to represent the energy in gravitational field (e.g., Watts and Moore, 2017).

The power spectrum of the spherical harmonic coefficients $\bar{C}_{n, m}^{s}$ implied by the topographic masses is:

$\sigma_{n}^{s}(r)=\left(\frac{R}{r}\right)^{n} \sqrt{\sum_{m=-n}^{+n}\left(\bar{C}_{n, m}^{s}\right)^{2}}$.

We calculated $\sigma_{n}^{s}$ by Eq. (25) at three distinct spherical surfaces: 1) slightly above the Brillouin sphere $(r=R+1 \mathrm{~m}), 2)$ at the reference sphere $\left(r=R_{0}\right)$, and 3$)$ at the sphere given by the minimum spherical radius of the topography $\left(r=\operatorname{MIN}\left[r^{\prime}\left(\varphi^{\prime}, \lambda^{\prime}\right)\right]\right)$, where $\operatorname{MIN}\left[r^{\prime}\left(\varphi^{\prime}, \lambda^{\prime}\right)\right]=$ $442049 \mathrm{~m}$ for 1 Ceres and $\operatorname{MIN}\left[r^{\prime}\left(\varphi^{\prime}, \lambda^{\prime}\right)\right]=1728277 \mathrm{~m}$ for the Moon.

Equation (24) specifies the power spectrum of the spheroidal harmonic coefficients $\bar{C}_{n, m}^{e}$. Correspondingly to the spherical case, we evaluated spheroidal power spectra at three different spheroidal surfaces, i.e.: 1) slightly above the oblate Brillouin spheroid $(u=b+1 \mathrm{~m})$, 2) at the oblate reference spheroid $(u=b)$, and 3$)$ at the oblate spheroid given by the minimum spheroidal coordinate $u$ determined from the DEMs $\left(u=\operatorname{MIN}\left[u^{\prime}\left(\beta^{\prime}, \lambda^{\prime}\right)\right]\right)$ and confocal with the oblate reference spheroid. We found the minimum values $\operatorname{MIN}\left[u^{\prime}\left(\beta^{\prime}, \lambda^{\prime}\right)\right]=438080 \mathrm{~m}$ for 1 Ceres and $\operatorname{MIN}\left[u^{\prime}\left(\beta^{\prime}, \lambda^{\prime}\right)\right]=1728217 \mathrm{~m}$ for the Moon.

Rigorously, harmonic series uniformly converge only above the Brillouin surfaces. Despite of this fact, harmonic expansions have been widely used for synthesising the functionals of the gravitational field below the respective fundamental surfaces, e.g., on the reference sphere, on the oblate reference spheroid, or on the topographic surface. Here, convergence cannot be guaranteed as extensively discussed for the spherical harmonic expansion of the gravitational field at the Earth's surface (e.g., Sjöberg, 1980; Colombo, 1983; Jekeli, 1983; Moritz, 1989, Sects. 6 and 7). Nevertheless, we intentionally selected the surfaces below the Brillouin ones, and within the range of the topographic heights, to identify possible divergence behaviour for the gravitational fields of 1 Ceres and of the Moon.

We note that the conventional spherical harmonic series have been modified such that they 
converge inside the Brillouin sphere (e.g., Takahashi and Scheeres, 2014). This has been achieved by expressing the gravitational potential as a function of spherical Bessel functions and spherical harmonic coefficients. However, any discussion or exploitation of these harmonic series is out of the scope of this study.

The square root of degree variances of the topographic gravitational field for the Moon are depicted in Fig. 4a. The spherical and spheroidal power spectra are indistinguishable when calculated at $r=R+1 \mathrm{~m}$ and at $u=b+1 \mathrm{~m}$ (black curves), and monotonically decrease. On the other hand, the power spectra calculated at the respective reference surfaces (red curves) increase above harmonic d/o 800. This is in agreement with (Hirt and Kuhn, 2017; Šprlák et al., 2018), who also reported the diverging behaviour of the spherical harmonic spectra inferred by the lunar topography at the reference sphere. Similarly, the power spectra diverge at $r=\operatorname{MIN}\left[r^{\prime}\left(\varphi^{\prime}, \lambda^{\prime}\right)\right]$ and at $u=\operatorname{MIN}\left[u^{\prime}\left(\beta^{\prime}, \lambda^{\prime}\right)\right]$ (green curves). However, the increase of the square root of the degree variances already starts at harmonic $\mathrm{d} / \mathrm{o} \sim 300$. We note that the rate of convergence/divergence is almost identical for both the spherical and the spheroidal representations in case of the lunar gravitational field.

Figure $4 \mathrm{~b}$ illustrates the square root of degree variances for 1 Ceres. The power spectra again monotonically decrease above the Brillouin surfaces (black curves) and diverge for the other surfaces (red and green curves). In contrast to the Moon, the spheroidal square root of degree variances start to increase at higher ds/os as compared to their spherical counterparts. For example, the spherical power spectrum at the reference sphere $r=R_{0}$ diverges from harmonic $\mathrm{d} / \mathrm{o} \sim 100$ (dotted red curve), while the same phenomenon can be seen for the spheroidal power spectrum at the oblate reference spheroid $u=b_{0}$ above harmonic d/o $\sim 270$ (solid red curve). Thus, the spheroidal spectra show an improved convergence rate with respect to its spherical equivalents. This proves an enhanced applicability of the spheroidal forward modelling approach for the oblate planetary body of 1 Ceres.

Alternatively to the examinations in the spectral domain, we also compared performance of the spherical and the spheroidal harmonic expansions in the spatial domain. We restricted to the gravitational fields implied by the highly-elliptical topographic masses of 1 Ceres. We synthesised the magnitude of the gravitational vector $\|\mathbf{g}\|$, which is invariant with respect to its mathematical representation. The spherical harmonic synthesis was carried out by the formula:

$$
\begin{aligned}
& \left\|\mathbf{g}^{s}\left(r, \Omega_{s}\right)\right\|=\left\|\nabla V\left(r, \Omega_{s}\right)\right\| \\
& =\left\{\left[\frac{\partial V\left(r, \Omega_{s}\right)}{\partial r}\right]^{2}+\frac{1}{r^{2}}\left[\frac{\partial V\left(r, \Omega_{s}\right)}{\partial \varphi}\right]^{2}+\frac{1}{r^{2} \cos ^{2} \varphi}\left[\frac{\partial V\left(r, \Omega_{s}\right)}{\partial \lambda}\right]^{2}\right\}^{1 / 2} .
\end{aligned}
$$

The gravitational potential in Eq. (26) was parametrised by the spherical harmonic series (Heiskanen and Moritz, 1967, Eq. 2-39):

$V\left(r, \Omega_{s}\right)=\frac{G M}{R} \sum_{n=0}^{\infty} \sum_{m=-n}^{+n}\left(\frac{R}{r}\right)^{n+1} \bar{C}_{n, m}^{s} \bar{Y}_{n, m}\left(\Omega_{s}\right)$,

where the coordinate triplet $r$ and $\Omega_{s}=(\varphi, \lambda)$ represents the planetocentric spherical coordinates of the computational point, i.e., the spherical radius $r \in[0, \infty)$, the spherical latitude $\varphi \in$ $\left[-90^{\circ},+90^{\circ}\right]$, and the spherical longitude $\lambda \in\left[0^{\circ},+360^{\circ}\right)$. As we assume that the spherical and the spheroidal coordinate triplets refer to the same planetocentric Cartesian coordinate system, longitudes in both curvilinear systems are identical. On the other hand, the spheroidal harmonic 

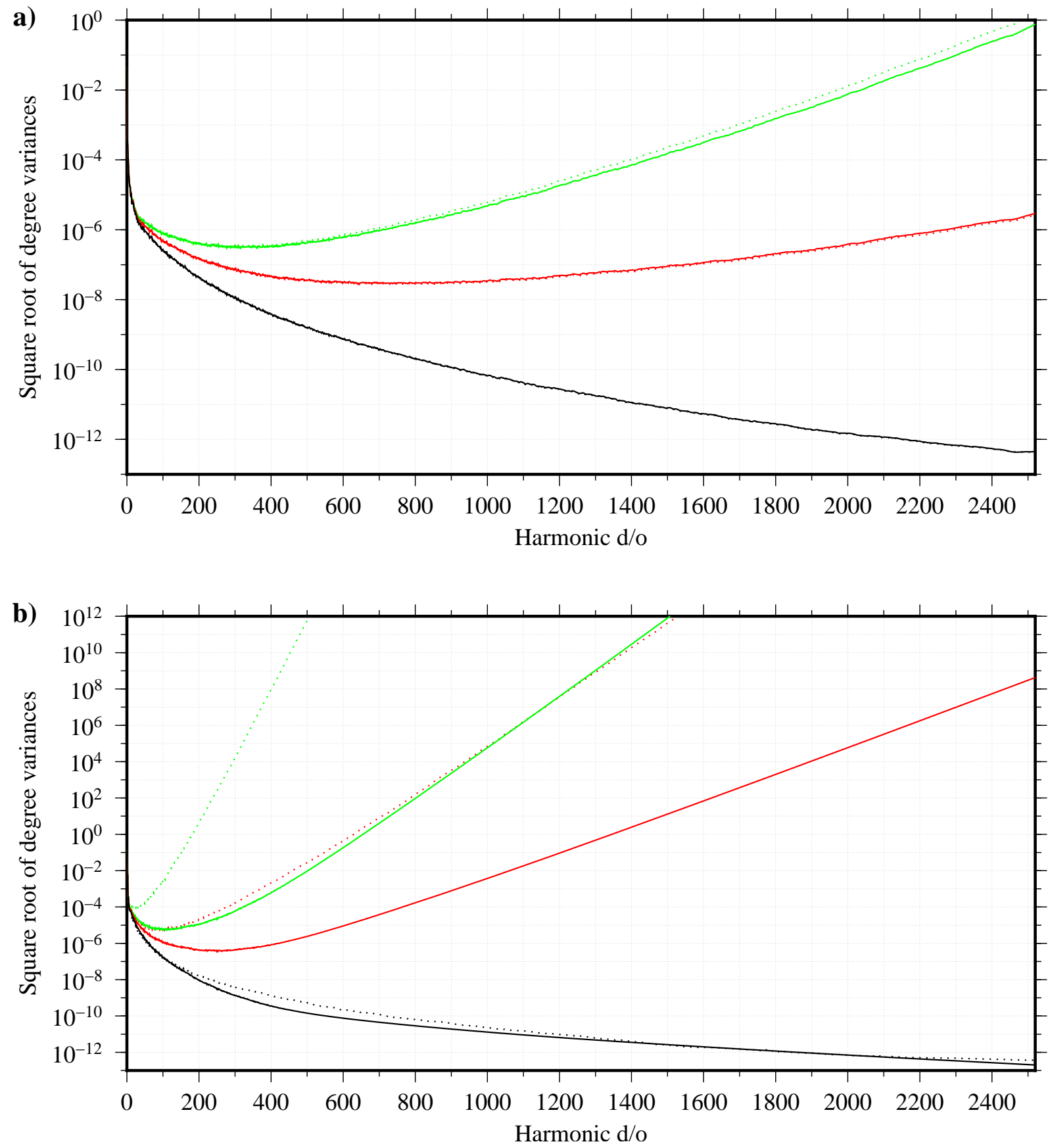

Figure 4: Square root of degree variances from the harmonic spectra for: a) the Moon and b) 1 Ceres. The spherical power spectra (dotted curves) $\sigma_{n}^{s}(r)$ were calculated for $r=R+1 \mathrm{~m}$ (black), $r=R_{0}$ (red), and $r=\operatorname{MIN}\left[r^{\prime}\left(\varphi^{\prime}, \lambda^{\prime}\right)\right]$ (green). The spheroidal power spectra (solid curves) $\sigma_{n}^{e}(u)$ were computed for $u=b+1 \mathrm{~m}$ (black), $u=b_{0}$ (red), and $u=\operatorname{MIN}\left[u^{\prime}\left(\beta^{\prime}, \lambda^{\prime}\right)\right]$ (green).

synthesis was performed by the expression:

$\left\|\mathbf{g}^{e}\left(u, \Omega_{e}\right)\right\|=\left\|\nabla V\left(u, \Omega_{e}\right)\right\|=\left\{\frac{u^{2}+\varepsilon^{2}}{u^{2}+\varepsilon^{2} \sin ^{2} \beta}\left[\frac{\partial V\left(u, \Omega_{e}\right)}{\partial u}\right]^{2}+\frac{1}{u^{2}+\varepsilon^{2} \sin ^{2} \beta}\left[\frac{\partial V\left(u, \Omega_{e}\right)}{\partial \beta}\right]^{2}\right.$ 
$\left.+\frac{1}{\left(u^{2}+\varepsilon^{2}\right) \cos ^{2} \beta}\left[\frac{\partial V\left(u, \Omega_{e}\right)}{\partial \lambda}\right]^{2}\right\}^{1 / 2}$,

where the gravitational potential is defined by the series of Eq. (5).

We calculated the magnitudes of the gravitational vector at the same computational point given by the coordinates $r=u=b+1 \mathrm{~m}, \varphi=\beta=90^{\circ}$, and $\lambda=0^{\circ}$, i.e., $1 \mathrm{~m}$ above the oblate Brillouin spheroid at the North pole. Thus, we considered a point with the largest deviation between the corresponding Brillouin surfaces. Here, uniform convergence is still guaranteed for the spheroidal harmonic expansion, while convergence of the spherical harmonic series is questionable.

Both harmonic syntheses were evaluated from d/o 0 up to the variable maximum $\mathrm{d} / \mathrm{o}$ $N_{\max } \in[0,2519]$. In such a way, the convergence/divergence can be tested for the whole spectral range. When synthesising the gravitational vector components at the poles or their proximity, singularities may occur due to the terms $1 / \cos \varphi$ and $1 / \cos \beta$ and due to the derivatives with respect to the variables $\varphi$ and $\beta$, see Eqs. (26) and (28). To avoid this problem, we implemented the non-singular expressions for the components of the gravitational vector (e.g., Eshagh, 2008). In addition, we used the X-number algorithm (Fukushima, 2012) for computing the associated Legendre functions of the first kind to avoid any numerical problems at ultra-high ds/os.

Common logarithm of $\|\mathbf{g}\|$ as a function of the harmonic d/o is depicted in Fig. 5. Values of this quantity are close for both the spherical (red curve) and the spheroidal (black curve) harmonic representations below $N_{\max } \approx 100$. Beyond d/o 100, however, the spherical expansion completely fails and provides unrealistically high values, e.g., by more than 60 orders of magnitude at d/o 2519. In contrast, the value of $\|\mathbf{g}\|$ by the spheroidal harmonic series is stable for all ds/os and does not show any signs of divergence.

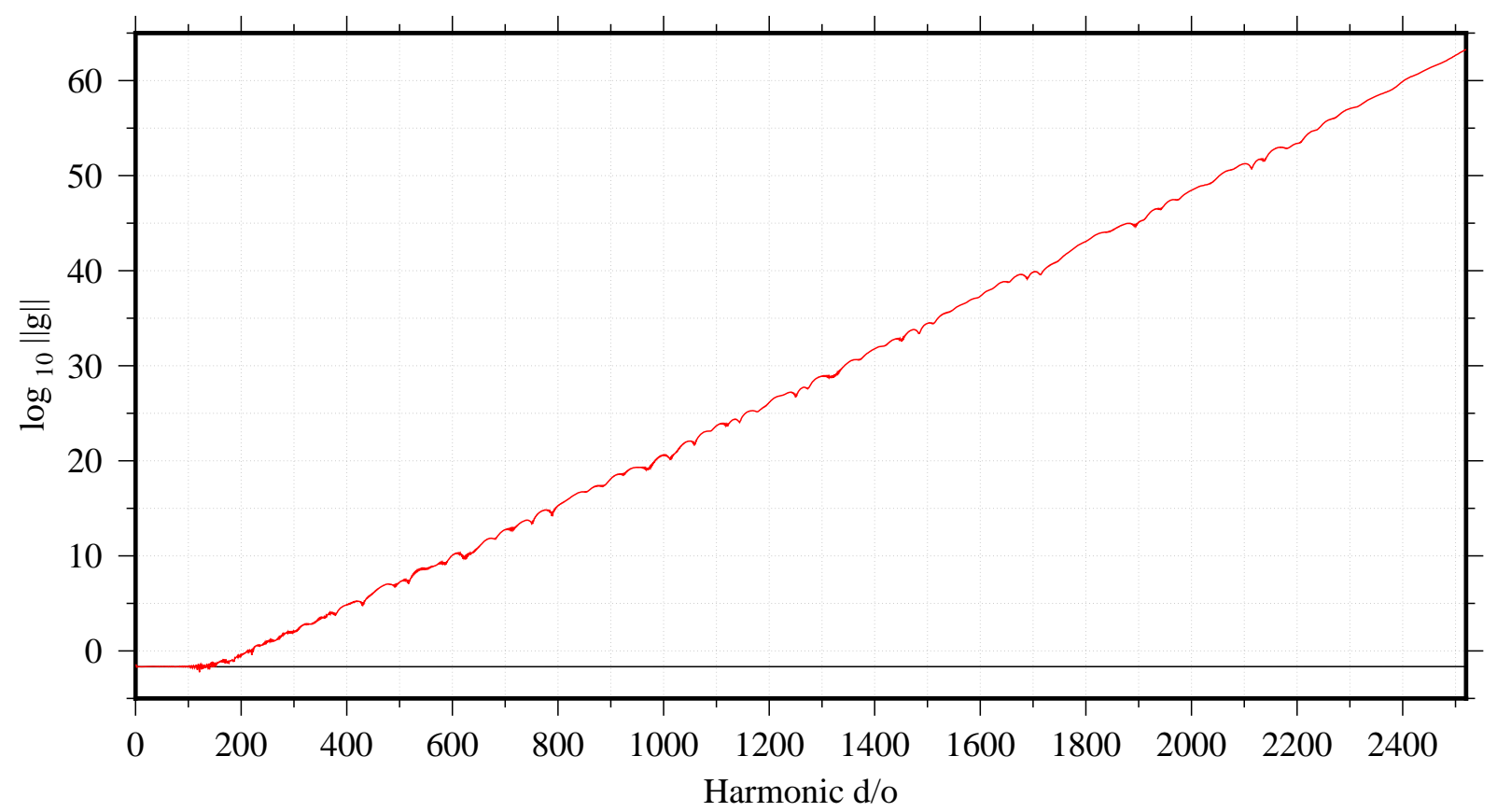

Figure 5: Common logarithm of the magnitude of the gravitational vector as synthesised by the spherical harmonic expansion of Eq. (26) (red) and by the spheroidal harmonic expansion of Eq. (28) (black).

Overall, our numerical experiments demonstrate an extended convergence space of the spheroidal 
harmonic expansion. This encourages for exploiting the spheroidal harmonic parametrisation and the spheroidal forward modelling in case of oblate planetary bodies like 1 Ceres. This conclusion is in conformity with the recent studies by (Hu and Jekeli, 2015; Sebera et al., 2016; Reimond and Baur, 2016), whose results also favour spheroidal (ellipsoidal) harmonic parametrisations to the simple spherical one for smaller planetary bodies, such as the Martian moons, asteroids Bennu and Castallia, or the comet 67P/Churyumov-Gerasimenko.

\section{Summary and Concluding Remarks}

A new method for forward modelling of global topographic gravitational fields is presented. Our so-called spheroidal forward modelling approach (Eq. (9)) is devoid of any postulates about the volumetric density and geometry of the gravitating body. We discretised the volume integral in Eq. (9) by finite spheroidal tesseroids that resulted in simple and numerically efficient recursions for the integrals of the associated Legendre functions of the first kind; see the Supplementary Material. We also presented a numerical algorithm in Eq. (21) that exploits the 1D discrete Fourier transform for more efficient computation, but the highest resolution models may require the use of a supercomputer.

Our algorithm was implemented in computer program and tested numerically. We considered two planetary bodies with contrasting flattening, namely the Moon $(f \approx 0.0003$, i.e., nearly spherical) and 1 Ceres $(f \approx 0.0747$, i.e., highly elliptical $)$. We calculated the spheroidal harmonic spectra up to high degree and order 2519 inferred by the homogeneous spheroidal shells and compared them with analytical spectra. This synthetic test confirmed high numerical accuracy, stability, and applicability of the numerical algorithm.

We also employed the spheroidal forward modelling and its spherical counterpart to calculate realistic global gravitational field models of the Moon and 1 Ceres. These realistic models were inferred by the homogeneous crusts of 1 Ceres and of the Moon and calculated up to degree and order 2519. The spherical and the spheroidal models were compared in the spectral and in the spatial domains. We showed that performance of both parametrisations was almost identical for the lunar gravitational field. On the other hand, we numerically proved an improved convergence rate when using the spheroidal parametrisation for the oblate planetary body of 1 Ceres. Thus, the spheroidal forward modelling and the corresponding harmonic representation should be preferred for oblate planetary bodies.

The new spheroidal forward modelling can be exploited by others for geophysical and planetary investigations (e.g., for calculating Bouguer anomalies, solving the inverse gravimetric problem, estimating crustal thickness and Moho depth or testing isostatic hypotheses), geodetic problems (e.g., for solving boundary value problems, geoid determination or upward/downward continuation of various gravitational field observables) or spacecraft navigation near highly elliptical planetary bodies. Parts of our spheroidal approach may even find application in other disciplines that deal with the analysis of elliptical geometries, such as in engineering or medicine (e.g., Dassios and Kariotou, 2003; Konstantinides et al., 2009; Kraiger and Schnizer, 2013).

The high-resolution gravitational field models of 1 Ceres and the Moon, and the computer program will be publicly available to potential users.

Acknowledgement: This research was supported financially by the Australian Government through the Australian Research Council's Discovery Projects funding scheme (project DP160104095). Digital elevation models are publicly available at the Planetary Data System or through (Preusker et al., 2016; Smith et al., 2010). The global gravitational field models will be publicly available at https://www.researchgate.net/ project/Understanding-the-Moons-crustal-structure-from-analysis-of-new-high -resolution-gravity-topo 
graphy-and-seismic-data. The computer program will be publicly available at https://sites.google.com/ site/michalsprlak/software. Thoughtful and constructive comments of the two anonymous reviewers are gratefully acknowledged. Thanks are also extended to the editor-in-chief Dr. Rosaly Lopes and the responsible editor Prof. Francis Nimmo for handling our manuscript.

\section{References}

Abramowitz M, Stegun IA (1972) Handbook of mathematical functions with formulas, graphs, and mathematical tables. Tenth Printing, National Bureau of Standards, Department of Commerce, Washington DC, U.S.A., 1046 pp.

Anderson EG (1976) The effect of topography on solutions of Stokes' problem. Unisurv Report No. S-14, School of Surveying, University of New South Wales, Kensington, Australia, 252 pp.

Ardalan AA (2000) High resolution regional geoid computation in the World Geodetic Datum 2000 based upon collocation of linearized observational functionals of the type GPS, gravity potential and gravity intensity. PhD. Thesis, Department of Geodesy and Geoinformatics, Stuttgart University, Stuttgart, Germany, 239 pp.

Ardalan AA, Karimi R (2014) Effect of topographic bias on geoid and reference ellipsoid of Venus, Mars, and the Moon. Celestial Mechanics and Dynamical Astronomy 118(1):75-88. https://doi.org/10.1007/s10569-013-9523-6.

Ardalan AA, Safari A (2004) Ellipsoidal terrain correction based on multi-cylindrical equal-area map projection of the reference ellipsoid. Journal of Geodesy 78(1-2):114-123. https://doi.org/10.1007/s00190-004-0381-6.

Asgharzadeh MF, von Frese RRB, Kim HR, Leftwich TE, Kim JW (2007) Spherical prism gravity effects by Gauss-Legendre quadrature integration. Geophysical Journal International 169(1):1-11. https://doi.org/10.1111/j.1365-246X.2007.03214.x.

Balmino G (1994) Gravitational potential harmonics from the shape of an homogeneous body. Celestial Mechanics and Dynamical Astronomy 60(3):331-364. https://doi.org/10.1007/BF00691901.

Balmino G, Lambeck K, Kaula WM (1973) A spherical harmonic analysis of the Earth's topography. Journal of Geophysical Research 78(2):478-481. https://doi.org/10.1029/JB078i002p00478.

Balmino G, Vales N, Bonvalot S, Briais A (2012) Spherical harmonic modelling to ultrahigh degree of Bouguer and isostatic anomalies. Journal of Geodesy 86(7):499-520. https://doi.org/10.1007/s00190-011-0533-4.

Burša M, Pěč K (1993) Gravity field and dynamics of the Earth. Springer-Verlag, Berlin Heidelberg, Germany, 333 pp.

Chandrasekhar S (1969) Ellipsoidal figures of equilibrium. The Silliman Foundation Lectures, Yale University Press, New Haven, Connecticut, U.S.A., 252 pp.

Cooley JW, Tukey JW (1965) An algorithm for the machine calculation of complex Fourier series. Mathematics of Computation 19(90):297-301. https://doi.org/10.1090/S0025-5718-19650178586-1. 
Colombo OL (1983) The convergence of the external spherical harmonic expansion of the gravitational potential. Bolletino di Geodesia e Scienze Affini 42(2):221-238.

Dassios G, Kariotou F (2003) Magnetoencephalography in ellipsoidal geometry. Journal of Mathematical Physics 44(1):220-241. https://doi.org/10.1063/1.1522135.

Deng X-L, Shen W-B (2018) Evaluation of gravitational curvatures of a tesseroid in spherical integral kernels. Journal of Geodesy 92(4):415-429. https://doi.org/10.1007/s00190-017-10733 .

D'Urso MG (2013) On the evaluation of the gravity effects of polyhedral bodies and a consistent treatment of related singularities. Journal of Geodesy 87(3): 239-252. https://doi.org/10.1007/s00190-012-0592-1.

Eshagh M (2008) Non-singular expressions for vector and gradient tensor of gravitation in a geocentric spherical frame. Computers \& Geosciences 34(12):1762-1768. https://doi.org/10.1016/j.cageo.2008.02.022.

Featherstone WE, Claessens SJ (2008) Closed-form transformation between geodetic and ellipsoidal coordinates. Studia Geophysica et Geodaetica 52(1):1-18. https://doi.org/10.1007/s11200-008-0002-6.

Fukushima T (2012) Numerical computation of spherical harmonics of arbitrary degree and order by extending exponent of floating point numbers. Journal of Geodesy 86(4):271-285. https://doi.org/10.1007/s00190-011-0519-2.

Fukushima T (2014) Numerical computation of spherical harmonics of arbitrary degree and order by extending exponent of floating point numbers: III integral. Computers \& Geosciences 63(1):17-21. https://doi.org/10.1016/j.cageo.2013.10.010.

Fukushima T (2018) Accurate computation of gravitational field of a tesseroid. Journal of Geodesy 92(12):1371-1386. https://doi.org/10.1007/s00190-018-1126-2.

Galassi M, Davies J, Theiler J, Gough B, Jungman G, Alken P, Booth M, Rossi F, Ulerich R (2013) GNU Scientific Library. Reference Manual, Edition 1.16, for GSL Version 1.13, 17 July 2013, 514 pp.

Garmier R, Barriot J-P (2001) Ellipsoidal harmonic expansions of the gravitational potential: Theory and application. Celestial Mechanics and Dynamical Astronomy 79(4):235-275. https://doi.org/10.1023/A:1017555515763.

Gil A, Segura J (1998) A code to evaluate prolate and oblate spheroidal harmonics. Computer Physics Communications 108(2-3):267-278. https://doi.org/10.1016/S0010-4655(97)00126-4.

Grafarend WE (2015) The reference figure of the rotating Earth in geometry and gravity space and an attempt to generalize the celebrated Runge-Walsh approximation theorem for irregular surfaces. International Journal on Geomathematics 6(1): 101-140. https://doi.org/10.1007/s13137-014-0068-y.

Grafarend WE, Engels J (1992) A global representation of ellipsoidal heights - geoid undulations or topographic heights - in terms of orthonormal functions. Part 1: "amplitude-modified" spherical harmonic functions. manuscripta geodaetica 17:52-58. 
Grafarend WE, Ardalan AA (1999) World Geodetic Datum 2000. Journal of Geodesy 73(11):611623. https://doi.org/10.1007/s001900050272.

Green G (1828) An essay on the application of mathematical analysis to the theories of electricity and magnetism. T. Wheelhouse, Nottingham, England, 72 pp.

Grombein T, Seitz K, Heck B (2016) The Rock-Water-Ice topographic gravity field model RWI_TOPO_2015 and its comparison to a conventional rock-equivalent version. Surveys in Geophysics 37(5):937-976. https://doi.org/10.1007/s10712-016-9376-0.

Grüninger W (1990) Zur topographisch-isostatischen Reduktion der Schwere. PhD. Thesis, Universität Karlsruhe, Karlsruhe, Germany.

Heck B, Seitz K (2007) A comparison of the tesseroid, prism and point-mass approaches for mass reductions in gravity field modelling. Journal of Geodesy 81(2):121-136. https://doi.org/10.1007/s00190-006-0094-0.

Heiskanen WA, Moritz H (1967) Physical geodesy. Freeman and Co., San Francisco, U.S.A., $364 \mathrm{pp}$.

Hirt C, Kuhn M (2017) Convergence and divergence in spherical harmonic series of the gravitational field generated by high-resolution planetary topography - a case study for the Moon. Journal of Geophysical Research - Planets 122(8):1727-1746. https://doi.org/10.1002/2017JE005298.

Hobson EW (1965) The theory of spherical and ellipsoidal harmonics. Chelsea Publishing Company, New York, U.S.A., 500 pp.

Hotine M (1969) Mathematical geodesy. ESSA Monograph No. 2, US Department of Commerce, Washington DC, U.S.A., 416 pp.

Hu X, Jekeli C (2015) A numerical comparison of spherical, spheroidal and ellipsoidal harmonic gravitational field models for small non-spherical bodies: examples for the Martian moons. Journal of Geodesy 89(2):159-177. https://doi.org/10.1007/s00190-014-0769-x.

Jekeli C (1981) The downward continuation to the Earth's surface of truncated spherical and ellipsoidal harmonic series of the gravity and height anomalies. Report No. 323, Department of Geodetic Science and Surveying, Ohio State University, Columbus, Ohio, U.S.A., 140 pp.

Jekeli C (1983) A numerical study of the divergence of spherical harmonic series of the gravity and height anomalies at the Earth's surface. Bulletin Géodésique 57(1-4):10-28. https://doi.org/10.1007/BF02520909.

Kellogg OD (1929) Foundations of potential theory. Verlag von Julius Springer, Berlin, Germany, $384 \mathrm{pp}$.

Konopliv AS, Park RS, Yuan D-N, Asmar SW, Watkins MM, Williams JG, Fahnestock E, Kruizinga G, Paik M, Strekalov D, Harvey N, Smith DE, Zuber MT (2013) The JPL lunar gravity field to spherical harmonic degree 660 from the GRAIL primary mission. Journal of Geophysical Research - Planets 118(7):1415-1434. https://doi.org/10.1002/jgre.20097. 
Konopliv AS, Park RS, Yuan D-N, Asmar SW, Watkins MM, Williams JG, Fahnestock E, Kruizinga G, Paik M, Strekalov D, Harvey N, Smith DE, Zuber MT (2014) High-resolution lunar gravity fields from the GRAIL Primary and Extended Missions. Geophysical Research Letters 41(5):1452-1458. https://doi.org/10.1002/2013GL059066.

Konopliv AS, Park RS, Vaughan AT, Bills BG, Asmar SW, Ermakov AI, Rambaux N, Raymond CA, Castillo-Rogez JC, Russell CT, Smith DE, Zuber MT (2018) The Ceres gravity field, spin pole, rotation period and orbit from the Dawn radiometric tracking and optical data. Icarus 299:411-429. https://doi.org/10.1016/j.icarus.2017.08.005.

Konstantinides JM, Mademlis A, Daras P, Mitkas PA, Strintzis MG (2009) Blind robust 3-D mesh watermarking based on oblate spheroidal harmonics. IEEE Transactions on Multimedia 11(1):23-38. http://dx.doi.org/10.1109/TMM.2008.2008913.

Kraiger M, Schnizer B (2013) Potential and field of a homogeneous magnetic spheroid of arbitrary direction in a homogeneous magnetic field in Cartesian coordinates. COMPEL - The international journal for computation and mathematics in electrical and electronic engineering 32(3):936-960. https://doi.org/10.1108/03321641311305845.

Kuhn M, Hirt C (2016) Topographic gravitational potential up to second-order derivatives: an examination of approximation errors caused by rock-equivalent topography (RET). Journal of Geodesy 90(9):883-902. https://doi.org/10.1007/s00190-016-0917-6.

Lachapelle G (1976) A spherical harmonic expansion of the isostatic reduction potential. Bolletino di Geodesia e Scienze Affini 35: 281-299.

Lee WHK, Kaula WM (1967) A spherical harmonic analysis of the Earth's topography. Journal of Geophysical Research 72(2):753-758. https://doi.org/10.1029/JZ072i002p00753.

Lowes FJ, Winch DE (2012) Orthogonality of harmonic potentials and fields in spheroidal and ellipsoidal coordinates: application to geomagnetism and geodesy. Geophysical Journal International 191(2):491-507. https://doi.org/10.1111/j.1365-246X.2012.05590.x.

Miller J (2019) Planetary spacecraft navigation. Space Technology Library, Vol. 37, Springer International Publishing, Cham, Switzerland, 390 pp. doi: https://doi.org/10.1007/978-3319-78916-3.

Mohr PJ, Taylor BN (2005) CODATA recommended values of the fundamental physical constants: 2002. Reviews of Modern Physics 77(1):1-107. https://link.aps.org/doi/10.1103/RevModPhys.77.1.

Moritz H (1989) Advanced physical geodesy. Second Edition. Wichmann, Karlsruhe, Germany, $500 \mathrm{pp}$.

Moritz H (1990) The figure of the Earth: theoretical geodesy and the Earth's interior. Wichmann, Karlsruhe, Germany, 279 pp.

Moon P, Spencer DE (1953) Recent investigations of the separation of Laplace's equation. Proceedings of the American Mathematical Society 4(2): 302-307. https://doi.org/10.2307/2031808.

Nagy D, Papp G, Benedek J (2000) The gravitational potential and its derivatives for the prism. Journal of Geodesy 74(7-8): 552-560. https://doi.org/10.1007/s001900000116. 
Nagy D, Papp G, Benedek J (2002) Corrections to: "The gravitational potential and its derivatives for the prism". Journal of Geodesy 76(8): 475. https://doi.org/10.1007/s00190-002-02647.

Neumann J (1848) Entwicklung der in elliptischen Koordinaten ausgedruckten reziproken Entfernung zweier Punkte in Reihen. Journal für die reine und angewandte Mathematik 37: 21-50.

Newton I (1687) Philosophiae Naturalis Principia Mathematica.

Novák P, Grafarend EW (2005) Ellipsoidal representation of the topographical potential and its vertical gradient. Journal of Geodesy 78(11-12):691-706. https://doi.org/10.1007/s00190-0050435-4.

Novák P, Šprlák M (2018) Spheroidal integral equations for geodetic inversion of geopotential gradients. Surveys in Geophysics 39(2):245-270. https://doi.org/10.1007/s10712-017-9450-2.

Park RS, Konopliv AS, Asmar SW, Bills BG, Gaskell RW, Raymond CA, Smith DE, Toplis MJ, Zuber MT (2014) Gravity field expansion in ellipsoidal harmonic and polyhedral internal representations applied to Vesta. Icarus 240:118-132. https://doi.org/10.1016/j.icarus.2013.12.005.

Pavlis NK, Rapp RH (1990) The development of an isostatic gravitational model to degree 360 and its use in global gravity modelling. Geophysical Journal International 100(3):369-378. https://doi.org/10.1111/j.1365-246X.1990.tb00691.x.

Pohánka V (1988) Optimum expression for computation of the gravity field of a homogeneous polyhedral body. Geophysical Prospecting 36(7): 733-751. https://doi.org/10.1111/j.13652478.1988.tb02190.x.

Pohánka V (2011) Gravitational field of the homogeneous rotational ellipsoidal body: a simple derivation and applications. Contributions to Geophysics and Geodesy 41(2):117-157. https://doi.org/10.2478/v10126-011-0005-0.

Preusker F, Scholten F, Matz K-D, Elgner S, Jaumann R, Roatsch T, Joy SP, Polanskey CA, Raymond CA, Russell CT (2016) Dawn at Ceres - Shape model and rotational state. 47th Lunar and Planetary Science Conference, 21-25 March 2016, The Woodlands, Texas, U.S.A, LPI Contribution No. 1903, p. 1954

Reimond S, Baur O (2016) Spheroidal and ellipsoidal harmonic expansions of the gravitational potential of small Solar System bodies. Case study: Comet67P/ChuryumovGerasimenko. Journal of Geophysical Research - Planets 121(3):497-515. https://doi.org/10.1002/2015JE004965.

Rexer M, Hirt C, Claessens S, Tenzer R (2016) Layer-based modelling of the Earth's gravitational potential up to 10-km scale in spherical harmonics in spherical and ellipsoidal approximation. Surveys in Geophysics 37(6):1035-1074. https://doi.org/10.1007/s10712-016-9382-2.

Roussel C, Verdun J, Cali J, Masson F (2015) Complete gravity field of an ellipsoidal prism by Gauss-Legendre quadrature. Geophysical Journal International 203(3):2220-2236. https://doi.org/10.1093/gji/ggv438.

Rummel R, Rapp RH, Sünkel H, Tscherning CC (1988) Comparisons of global topographic/isostatic models to the Earth's observed gravity field. Report No. 388, Department of Geodetic Science and Surveying, Ohio State University, Columbus, Ohio, U.S.A., 33 pp. 
Sebera J, Bezděk A, Pešek I, Henych T (2016) Spheroidal models of the exterior gravitational field of Asteroids Bennu and Castalia. Icarus 272:70-79. https://doi.org/10.1016/j.icarus.2016.02.038.

Sjöberg LE (1980) On the convergence problem for the spherical harmonic expansion of the geopotential at the surface of the Earth. Bolletino di Geodesia e Scienze Affini 39(3):261-270.

Smith DE, Zuber MT, Neumann GA, Lemoine FG, Mazarico E, Torrence MH, McGarry JF, Rowlands DD, Head III JW, Duxbury TH, Aharonson O, Lucey PG, Robinson MS, Barnouin OS, Cavanaugh JF, Sun X, Liiva P, Mao D-D, Smith JC, Bartels AE (2010) Initial observations from the Lunar Orbiter Laser Altimeter (LOLA). Geophysical Research Letters 37(18): L18204. https://doi.org/10.1029/2010GL043751.

Šprlák M, Han S-C, Featherstone W (2018) Forward modelling of global gravity fields with 3D density structures and an application to the high-resolution $(\sim 2 \mathrm{~km})$ gravity fields of the Moon. Journal of Geodesy 92(8):847-862. https://doi.org/10.1007/s00190-017-1098-7.

Takahashi Y, Scheeres DJ (2014) Small body surface gravity fields via spherical harmonic expansions. Celestial Mechanics and Dynamical Astronomy 119(2): 169-206. https://doi.org/doi:10.1007/s10569-014-9552-9.

Tenzer R, Hirt C, Novák P, Pitoňák M, Šprlák M (2016) Contribution of mass density heterogeneities to the quasigeoid-to-geoid separation. Journal of Geodesy 90(1):65-80. https://doi.org/10.1007/s00190-015-0858-5.

Thong NC, Grafarend WE (1989) A spheroidal harmonic model of the terrestrial gravitational field. manuscripta geodaetica 14:285-304.

Uieda L, Barbosa VCF, Braitenberg C (2016) Tesseroids: Forward-modelling gravitational field in spherical coordinates. Geophysics 81(5): F41-F48. https://doi.org/10.1190/geo2015-0204.1.

Wang WX (1988) The potential for a homogeneous spheroid in a spheroidal coordinate system: I. At an exterior point. Journal of Physics A: Mathematical and General 21(22):4245-4250. https://doi.org/10.1088/0305-4470/21/22/026.

Wang YM, Yang X (2013) On the spherical and spheroidal harmonic expansion of the gravitational potential of the topographic masses. Journal of Geodesy 87(10-12):909-921. https://doi.org/10.1007/s00190-013-0654-z.

Watts AB, Moore JDP (2017) Flexural isostasy: Constraints from gravity and topography power spectra. Journal of Geophysical Research - Solid Earth 122(10):8417-8430. https://doi.org/10.1002/2017JB014571.

Werner RA (2017) The solid angle hidden in polyhedron gravitation formulations. Journal of Geodesy 91(3): 307-328. https://doi.org/10.1007/s00190-016-0964-z.

Werner RA, Scheeres DJ (1996) Exterior gravitation of a polyhedron derived and compared with harmonic and mascon gravitation representations of asteroid 4769 Castalia. Celestial Mechanics and Dynamical Astronomy 65(3): 313-344. https://doi.org/10.1007/BF00053511.

Wessel P, Smith WHF, Scharroo R, Luis JF, Wobbe F (2013) Generic Mapping Tools: Improved version released, EOS - Transactions of the AGU 94(45):409-410. https://doi.org/10.1002/2013EO450001. 
Wieczorek MA (2007) Gravity and topography of the terrestrial planets. Treatise on Geophysics, Vol. 10, Elsevier, Amsterdam, The Netherlands, pp. 165-206. https://doi.org/10.1016/B978044452748-6/00156-5.

Wieczorek MA, Neumann GA, Nimmo F, Kiefer WS, Taylor GJ, Melosh HJ, Phillips RJ, Solomon SC, Andrews-Hanna JC, Asmar SW, Konopliv AS, Lemoine FG, Smith DE, Watkins MM, Williams JG, Zuber MT (2013) The crust of the Moon as seen by GRAIL. Science 339(6120):671-675. https://doi.org/10.1126/science.1231530.

Wieczorek MA, Phillips RJ (1998) Potential anomalies on a sphere: applications to the thickness of the lunar crust. Journal of Geophysical Research - Planets 103(E1):1715-1724. https://doi.org/10.1029/97JE03136.

Wild-Pfeiffer F (2008) A comparison of different mass elements for use in gravity gradiometry. Journal of Geodesy 82(10):637-653. https://doi.org/10.1007/s00190-008-0219-8. 